\title{
The Jamming Avoidance Response in Eigenmannia Is Controlled by Two Separate Motor Pathways
}

\author{
Walter Metzner \\ University of California at San Diego, Neurobiology Unit, Scripps Institution of Oceanography, La Jolla, California \\ 92093-0202 and University of California at Riverside, Department of Biology, Riverside, California 92521-0427
}

The gymnotiform fish Eigenmannia generates weakly electric signals for electrolocation and communication. The signals are produced by electric organ discharges (EODs) that are driven by a medullary pacemaker nucleus. To avoid jamming by neighboring conspecifics with similar frequencies, a fish raises its own EOD frequency if the neighbor's frequency is lower, and it lowers its EOD frequency if the neighbor's frequency is higher (Watanabe and Takeda, 1963). Both the raising and lowering of EOD frequency of this jamming avoidance response (JAR; Bullock et al., 1972) are thought to be controlled by feature-extracting neurons in the diencephalic prepacemaker nucleus (PPn-G) that discriminate the sign of the frequency difference between the jamming signal and the fish's EOD (Kawasaki et al., 1988a; Rose et al., 1988; for review, see Heiligenberg, 1991). These prepacemaker neurons are excited in response to lower jamming frequencies, thereby raising the frequency, and inhibited by higher jamming frequencies, producing a discharge deceleration. The results of experiments presented here, however, suggest a mechanism for the motor control of the JAR that is different from the one described previously (see, e.g., Heiligenberg, 1991).

Two prepacemaker nuclei, one PPn-G and one sublemniscal prepacemaker nucleus (SPPn) (Keller et al., 1991a,b), which provide the only known inputs to the pacemaker, were lesioned selectively. This article explores the effects of these lesions on the JAR. Pharmacological experiments were used to elucidate the transmitter types involved. The results suggest that the JAR is controlled by two separate motor pathways. One controls frequency rises and originates in the dorsal substructure of the nucleus electrosensorius (Keller, 1988). It sends excitatory connections to the diencephalic prepacemaker and finally to the pacemaker nucleus, where AMPA-type receptors mediate the synaptic transmission. The second pathway controls frequency decreases and originates in the ventral substructure of the nucleus electrosensorius. It provides GABAergic input to the SPPn. The SPPn

\footnotetext{
Received Aug. 26, 1992; revised Oct. 27, 1992; accepted Nov. 3, 1992.

I thank Walter Heiligenberg, Kip Keller, Masashi Kawasaki, Carl Hopkins, Svenja Viete, Mark Konishi, Ted Bullock, John Spiro, Brooke Armstrong, and the anonymous reviewers for most helpful comments and suggestions. Grace Kennedy and Georgia Malan provided excellent technical support. This work was supported by Grants DFG Me953/1-1 and UCR 19900 NS7B to W.M. and Grants R37 MH26149-15, NINCDS R01 NS22244-06, and NSF 5-8716781 to Walter Heiligenberg.

Correspondence should be addressed to Walter Metzner, University of California, Department of Biology, Riverside, CA 92521-0427.

Copyright (C) 1993 Society for Neuroscience $0270-6474 / 93 / 131862-17 \$ 05.00 / 0$
}

is tonically active and also controls the EOD frequency even in the absence of jamming signals. Its projection to the pacemaker nucleus is mediated by NMDA-type receptors.

The results of this study suggest that there is no single population of final, feature-extracting elements or "recognition units" that controls JAR-related shifts of the pacemaker frequency. Instead, the motor control of the JAR consists of an interaction of two independent pathways according to a "push-pull" principle.

[Key words: electric fish, electrolocation, sensorimofor integration, push-pull principle, pacemaker control, lesion studies, pharmacology]

Gymnotiform fish generate weakly electric fields for electrolocation and communication purposes. The electric signals are produced by discharges of an electric organ that is located in the tail section of their body. The electric organ discharges (EODs) are driven by a medullary pacemaker nucleus that triggers each discharge cycle. In the gymnotiform fish Eigenmannia, EODs are continual, nearly sinusoidal signals with a fundamental frequency ranging between 150 and $500 \mathrm{~Hz}$. The otherwise constant EOD frequency (equals resting frequency) can be modulated in the context of communication, such as courtship or aggression (see review by Hopkins, 1988). In addition, Eigenmannia shifts its EOD frequency to avoid jamming by signals that originate from neighboring conspecifics (Watanabe and Takeda, 1963). This jamming avoidance response (JAR; Bullock et al., 1972) can provide a separation of EOD frequencies among neighboring fish that is required for accurate electrolocation of objects (Heiligenberg, 1973). The JAR functions even in temporarily immobilized animals, which allows one to study the neuronal implementation of this behavior in electrophysiological experiments in awake, behaving animals (Scheich and Bullock, 1974). Therefore, these fish provide an excellent system in which the neuronal networks that control animal behavior can be analyzed from the sensory periphery to the motor output.

Many behavioral experiments have determined the computational rules that guide the JAR in Eigenmannia (for a most recent review, see Heiligenberg, 1991; Heiligenberg and Kawasaki, 1992). Numerous subsequent neurophysiological and anatomical studies have revealed many elements of the neuronal implementation of the JAR (Heiligenberg, 1991).

Eigenmannia raises its EOD frequency in response to jamming signals of slightly lower frequency and lowers its EOD frequency in response to signals of slightly higher frequency. The fish do not use an internal "corollary" signal of their own EOD to determine whether a jamming signal is lower or higher in frequency (Bullock et al., 1972; Heiligenberg et al., 1978). 
Instead, they evaluate modulations in the phase and amplitude of their own signal combined with the interfering signal to discriminate the sign of the frequency difference (see Heiligenberg, 1991). Phase and amplitude information are analyzed separately at the level of the rhombencephalon, but this information converges within the dorsal torus semicircularis of the fish's midbrain to yield "sign-selective" neurons that discriminate the sign of the frequency difference (Bastian and Heiligenberg, 1980a,b; Heiligenberg and Rose, 1985; Rose and Heiligenberg, 1985a). However, the spatial and temporal resolution of toral neurons is still insufficient to explain all aspects of the behavior (Rose and Heiligenberg, 1985a,b, 1986; Carr et al., 1986a,b; Heiligenberg and Rose, 1986; for review, see Heiligenberg, 1991). A higher stimulus sensitivity and temporal resolution is achieved in subsequent structures that also generate the motor command that finally controls the JAR. The current concept of the motor control of the JAR is presented schematically in Figure 1.

The torus semicircularis dorsalis is known to project to the diencephalic complex of the nucleus electrosensorius (Carr et al., 1981) where sign-selective responses were demonstrated by extracellular (Bastian and Yuthas, 1984; Keller, 1988) and intracellular recordings (Heiligenberg et al., 1991). The temporal and spatial resolution of some of these neurons is almost as accurate as that found at the behavioral level. Keller and Heiligenberg (1989) used iontophoretic application of L-glutamate to define distinct areas within the nucleus electrosensorius. Stimulation of a dorsal area (Fig. 1, nE ) causes a smooth rise in EOD frequency, while stimulation of a more ventrally located area (Fig. 1, nE $\downarrow$ ) causes a smooth decline in EOD frequency. Selective lesions of these areas eliminate the corresponding frequency shifts from the JAR (Keller, 1988). Thus, the dorsal and the ventral nucleus electrosensorius appear to be necessary for the control of the JAR.

Until recently, the only known structure providing input to the pacemaker nucleus was the diencephalic prepacemaker nucleus (PPn) (Heiligenberg et al., 1981; Szabo et al., 1989), which as extracellular iontophoretic injections of L-glutamate have shown is composed of two functionally distinct subnuclei, a medial portion where L-glutamate elicits gradual accelerations of the pacemaker frequency, therefore termed PPn-G, and a more lateral and ventral portion where L-glutamate or intracellular microstimulations elicit abrupt frequency modulations or "chirps," therefore called PPn-C (Kawasaki and Heiligenberg, 1988; Kawasaki et al., 1988a). The gradual frequency rises are similar to those occurring during the JAR, whereas chirps are signals used during courtship and aggression (Hopkins, 1974a,b, 1988; Hagedorn and Heiligenberg, 1985). In accordance with the results from glutamate injections into the PPn$\mathrm{G}$, extracellular recordings in the vicinity of this nucleus reveal sign-selective neurons that are excited by negative frequency differences and slightly inhibited by positive frequency differences (Rose et al., 1988). Their spatial and temporal resolution is often as accurate as that observed at the behavioral level (Rose and Heiligenberg, 1985b; Kawasaki et al., 1988b). Small lesions of the PPn caused a reduction of the JAR, while larger lesions including laterally adjacent areas eliminated the JAR completely but also caused damage to the animal's ability to maintain normal body posture (Rose et al., 1988; M. Kawasaki, personal communication). These results suggested that neurons within the PPn-G represent the final, feature-extracting elements that process sensory information for the control of JAR-related shifts of the pacemaker frequency (Kawasaki et al., 1988; Rose et al.,

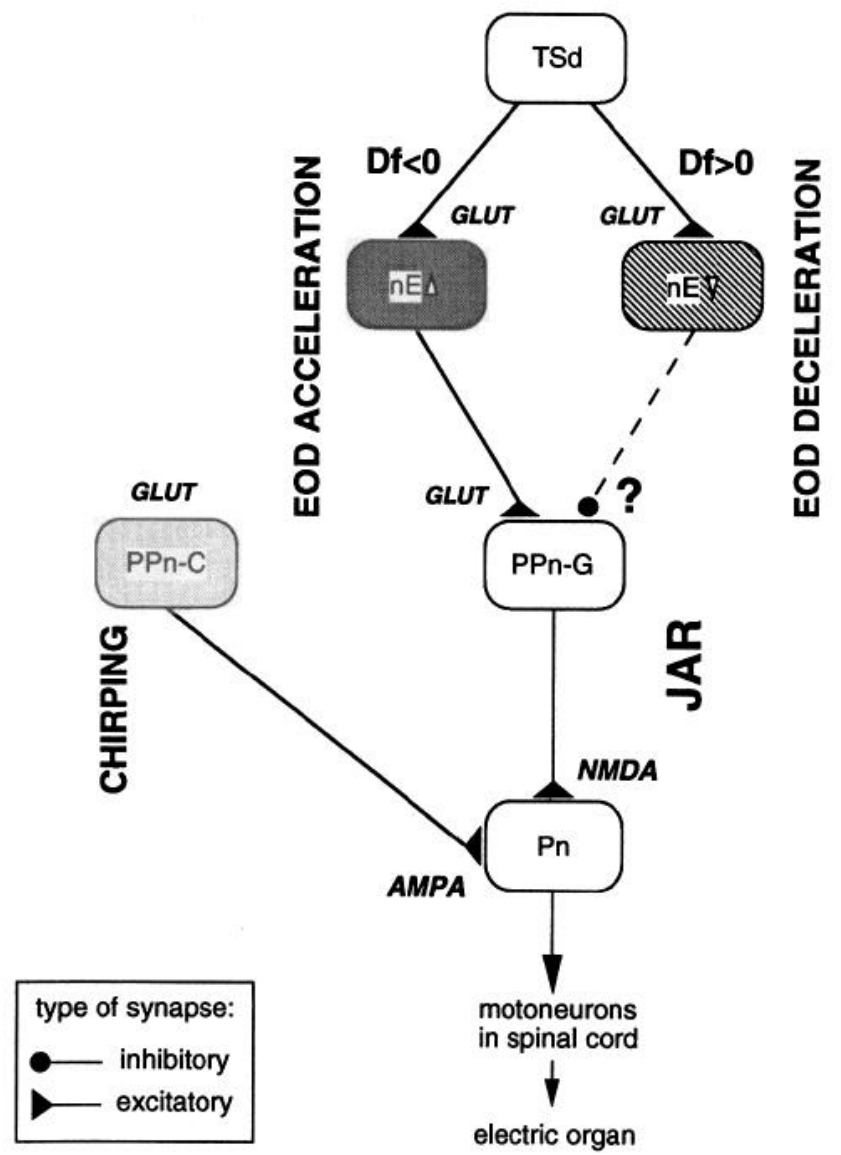

Figure 1. Original hypothesis of the motor control of the JAR in Eigenmannia (Kawasaki et al., 1988a,b; Rose et al., 1988; Dye et al., 1989; Keller and Heiligenberg, 1989; Heiligenberg, 1991). The torus semicircularis dorsalis (TSd) contains two classes of "sign-selective" cells that are excited either by positive or by negative frequency differences $(D f)$ between the jamming signal and the fish's own EOD. The dorsal portion of the nucleus electrosensorius $(n E\rceil)$ contains higherorder sign-selective neurons, excited by negative frequency differences. Its stimulation accelerates the pacemaker. Higher-order sign-selective cells that are excited by positive frequency differences are located in the ventral portion of the nucleus electrosensorius $(n E \downarrow)$. Its stimulation lowers the EOD frequency. The PPn-G represents the top of a neuronal hierarchy controlling the EOD frequency. Its neurons are excited by negative frequency differences and inhibited by positive frequency differences. Their excitation raises and their inhibition lowers the frequency of the pacemaker nucleus $(P n)$, which triggers each discharge cycle of the electric organ by a single command pulse. Chirp-like frequency modulations are generated by the PPn-C and mediated by AMPA receptors. The effect of the PPn-G/CP was assumed to be mediated by NMDA-type receptors. Compare with Figure 2, which incorporates the new findings about the motor control of the JAR. GLUT indicates where electrophoretic injection of $\mathrm{L}$-glutamate elicits effects.

1988; Heiligenberg, 1991). The excitation of these "recognition units" in the PPn-G due to negative frequency differences raises the pacemaker frequency, and the inhibition of these prepacemaker neurons in response to positive frequency differences lowers it (see Fig. 1).

The results of the study presented here indicate, however, that there is no single class of final recognition units. Instead, the control of the JAR is mediated by two separate motor pathways that run from the diencephalic nucleus electrosensorius to the medullary pacemaker nucleus (Metzner and Heiligenberg, 1991b; Metzner, 1992). This is schematically shown in Figure 2 (compare with Fig. 1). One pathway originates in the dorsal portion 


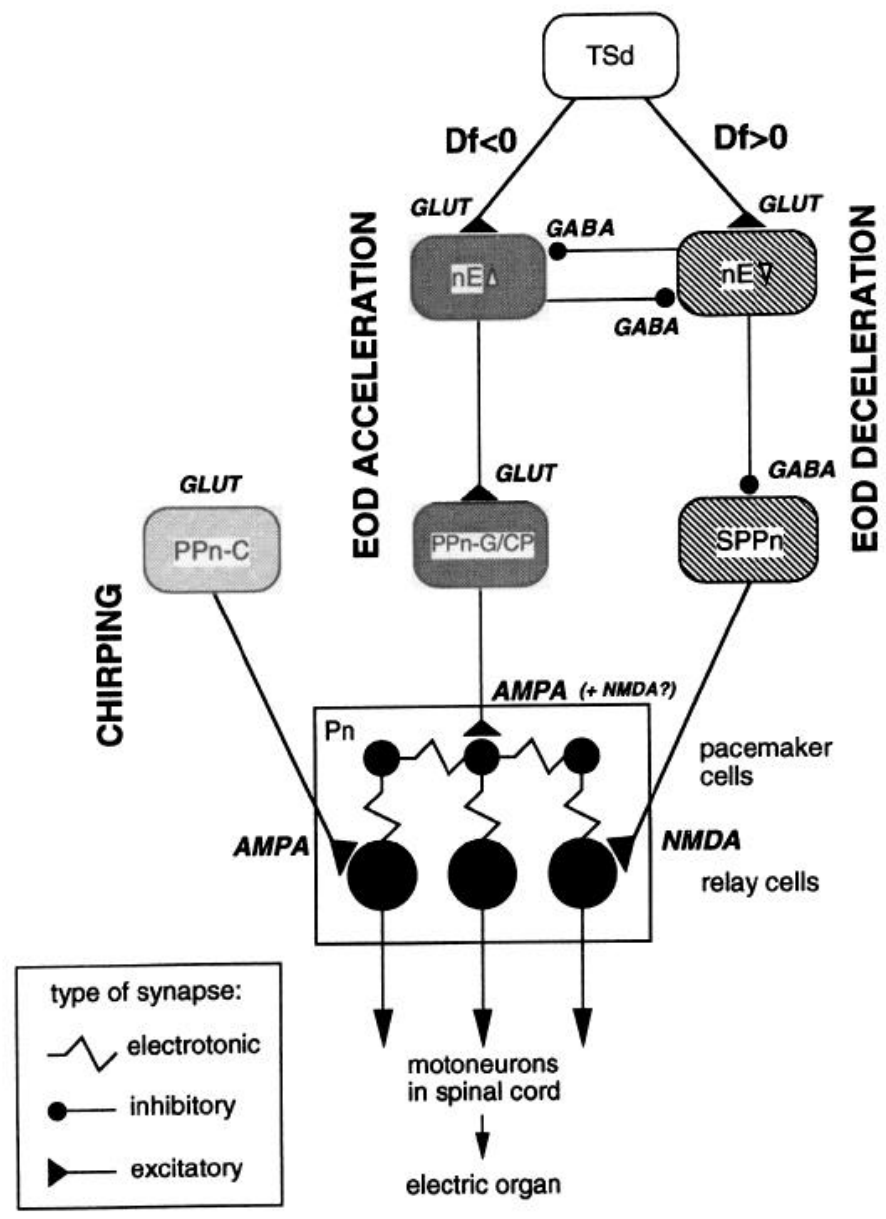

Figure 2. Present hypothesis of the motor control of the JAR in Eigenmannia. This flow diagram is explained in more detail in the Discussion section. Compare with Figure 1, which shows the original hypothesis. The response to jamming stimuli with negative frequency difference $(D f)$, that is, EOD accelerations, is controlled by a purely excitatory pathway (darkly stippled structures) that originates in the dorsal nucleus electrosensorius ( $n E \uparrow)$, passes via the PPn-G/CP, and is assumed to terminate on pacemaker $(P n)$ cells, where the synaptic transmission is mediated primarily by AMPA-type receptors. The pathway that controls the response to positive frequency differences, that is, EOD decelerations (hatched structures), begins in the ventral nucleus electrosensorius $(n E \mid)$ and continues via GABAergic connections within the SPPn. The SPPn projects to relay cells, where NMDA-type receptors mediate synaptic transmission. This second pathway also controls the resting frequency by the tonic activity of the SPPn. Communication signals (chirps) are generated by the PPn-C (lightly stippled), and its connection with relay cells in the pacemaker nucleus via AMPA-type receptors (Kawasaki et al., 1988a; Dye et al. 1989; Kawasaki and Heiligenberg, 1990; verified also by my own experiments). The reciprocal GABAergic connection between the dorsal $(n E \dagger)$ and ventral nucleus electrosensorius $(n E l)$ was demonstrated by Keller and Heiligenberg (1989). GLUT indicates where electrophoretic injection of L-glutamate elicits effects (Kawasaki et al., 1988a; Keller and Heiligenberg, 1989; Kawasaki and Heiligenberg, 1990).

of the nucleus electrosensorius (nE†) and involves the PPn-G. This tract controls the increase of the pacemaker frequency in response to negative frequency differences. The second motor pathway starts in the ventral portion of the nucleus electrosensorius (nE $]$ ) and includes the sublemniscal prepacemaker nucleus (SPPn) in the mesencephalon. This additional source of input to the pacemaker nucleus was only recently discovered (Keller et al., 1991a,b). The tract via the SPPn controls the lowering of the pacemaker frequency in response to positive frequency differences. The two motor pathways finally converge at the level of the pacemaker nucleus, where they interact according to a "push-pull" principle.

\section{Materials and Methods}

Thirty-seven Eigenmannia from 12 to $20 \mathrm{~cm}$ in body length were used in this study. All fish had been bred and raised in the laboratory. They were immobilized by intramuscular injection of Flaxedil (gallamine triethiodide; less than $5 \mu \mathrm{g} / \mathrm{gm}$ body weight) and gently suspended in the center of the experimental aquarium (resistivity, 9-12 $\mathrm{k} \Omega \cdot \mathrm{cm}, 26$ $28^{\circ} \mathrm{C}$ ) by a foam-lined forceps with only the dorsal surface of their head protruding above the water surface. They were respirated with a stream of aquarium water via a glass tube inserted in their mouth. Under local anesthesia (Novocain), a small Plexiglas rod was glued to the parietal bone to stabilize the fish further. Although Flaxedil strongly attenuated the EODs, residual signals ( $50 \mu \mathrm{V}$ to $1 \mathrm{mV}$ ), locked to the spinal command cells, could still be monitored with a suction electrode fitted over the tip of the tail. These signals were amplified (Grass P-15) and either stored on tape (HP 3964A) or fed directly to a computer for frequency analysis. The EOD frequency was monitored continually on a chart recorder.

Since the curarization attenuated the animal's EOD to a subthreshold level, the JAR could be elicited exclusively by artificial stimuli (Scheich and Bullock, 1974). The fish's EOD was mimicked by a sinusoidal stimulus $\left(\mathrm{S}_{1}\right)$ applied between an electrode in the mouth and an electrode near the tip of the tail. Frequency and amplitude of the EOD mimic were adjusted to those of the EOD before curarization $(150-400 \mathrm{~Hz}$, 1-3 $\mathrm{mV} / \mathrm{cm}$ near and perpendicular to the head surface). A second sinusoidal stimulus $\left(\mathrm{S}_{2}\right)$, mimicking the jamming signal of a neighbor, could be delivered through any pair of diagonally opposed electrodes in a quasicircular array surrounding the fish. The second jamming signal could also be applied through the same pair of electrodes mimicking the fish's own EOD by electronically adding the two signals. In this "identical geometry" condition, modulations of differential phase are absent, and JARs cannot be elicited (Heiligenberg et al., 1978). The intensity of $\mathrm{S}_{2}$ was normally $30 \%$ of that of $\mathrm{S}_{1}$, measured near the body surface of the fish and perpendicular to its head. The stimulus electrode was oriented perpendicular to the fish's body axis. The magnitude of the frequency difference between $S_{2}$ and $S_{1}$ was set at the fish's optimal value for eliciting a JAR, typically $\pm 4 \mathrm{~Hz}$.

Previous studies of the JAR have used a stimulation regime in which the sign of the frequency difference between $S_{1}$ and $S_{2}$ was continuously alternated between positive and negative values. Under these experimental conditions, it is impossible to separate the response to either sign of the frequency difference from the recovery of the response from the preceding cycle. Recovery phenomena, however, are of particular importance if the JAR is to be studied before and after a lesion of a presumptive neural circuit. I examined the recovery time course in two ways: either jamming signals with only one sign of frequency difference were presented repeatedly; or within a series of alternating positive and negative frequency differences, the jamming stimulus was turned off just before the frequency difference switched to the opposite sign. The resulting recovery time courses were compared with the time courses of EOD frequency changes to either sign of frequency difference. The nearly exponential time courses of EOD frequency changes were determined by calculating the time constant, $\alpha$, according to $0.5=\exp \left(-\alpha T_{y_{2}}\right)$, with $T_{1 / 2}$ being the time when the value of the JAR-related frequency shift had relaxed $50 \%$. After time $T_{1 / 2}$, the EOD frequency has changed to $1 / e$ of its original value. Therefore, the slower the EOD frequency changes, that is, the larger $T_{1 / 2}$ is, the smaller is the corresponding value of $\alpha$.

The diencephalic prepacemaker nucleus, the nucleus electrosensorius, and the sublemniscal prepacemaker nucleus were reached by removing frontal bone overlying the rostral optic tectum and cerebellar vermis (approximately $2 \mathrm{~mm}^{2}$ ) under local anesthesia (Novocain). In some cases a larger hole (ca. $2 \times 4 \mathrm{~mm}$ ) was required to expose both tecta to allow bilateral penetrations. The prepacemaker nucleus is located approximately $2100 \mu \mathrm{m}$ underneath the tectal commissure. It was localized by iontophoretic injection of $\mathrm{L}$-glutamate (Kawasaki et al., 1988a). The nucleus electrosensorius is located approximately $400-500 \mu \mathrm{m}$ lateral and $100-300 \mu \mathrm{m}$ dorsal to the chirp-producing portion of the diencephalic prepacemaker (PPn-C) (Keller, 1988; Keller et al., 1990). The location of the sublemniscal prepacemaker nucleus is approximately $400-500$ $\mu \mathrm{m}$ caudal to the diencephalic prepacemaker nucleus. The pacemaker 
nucleus was reached by making a small slit in the cartilaginous tissue above the corpus cerebelli. The center of the medullary pacemaker nucleus could be localized by detecting its field potential, which is phaselocked to the EOD, using a $3 \mathrm{~m} \mathrm{NaCl}$-filled glass pipette with an inner tip diameter of a few micrometers.

Stimulating and iontophoresing electrodes were triple-barrel glass micropipettes. Electrodes were variously filled with $\mathrm{L}$-glutamate for stimulation, $\mathrm{NaCl}$ for field potential recordings of the pacemaker, indium for lesioning or electrical stimulation, or different transmitter agonists and antagonists, respectively. Cell clusters were stimulated by localized iontophoresis of $\mathrm{L}$-glutamate [Sigma; $0.1 \mathrm{~m}$ in water, $\mathrm{pH} \mathrm{8}$, ca. $100 \mathrm{nA}$ (negative DC), with an inner tip diameter of the electrode of $<10 \mu \mathrm{m}$ ] For electrical stimulation one barrel was filled with indium and trains of $200 \mathrm{~Hz}$ electric pulses of $1-15 \mu \mathrm{A}$, approximately $1 \mathrm{msec}$ in duration were applied (World Precision Instruments Accupulser A310). For lesioning brain tissue, a high-frequency current (Birtcher Hyfrecator 733, bipolar setting between 30 and 40 for 1-2 sec; see Keller and Heiligenberg, 1989) was applied through the indium-filled barrel. The resulting lesions had a diameter of $100-150 \mu \mathrm{m}$. The site of the lesion was later verified in histological sections of the brain. Stimulation sites were marked by lesions or by iontophoretic injection of Alcian blue (Sigma; $2 \%$ in acetate-acetic acid buffer, $\mathrm{pH} 4.0$ ) from another barrel of the same micropipette assembly. A positive current of 2-8 $\mu \mathrm{A}$ DC yielded a discrete blue spot (ca. $20-40 \mu \mathrm{m}$ diameter) in histological sections. In some cases the stimulation sites were also verified by injection of the neurotracer choleratoxin (List Biological Laboratories) through one of the three barrels of the microelectrode assembly (pressure injection; see below).

The pharmacological agents tested were GABA ( $\gamma$-amino- $n$-butyric acid; Sigma; $0.5 \mathrm{M}$ in water, $\mathrm{pH} 3.5$ with $\mathrm{HCl}$ ); the $\mathrm{GABA}_{\mathrm{A}}$ antagonist bicuculline methiodide (Sigma; $2 \mathrm{~mm}$ in $165 \mathrm{~mm} \mathrm{NaCl}, \mathrm{pH} \mathrm{3.2)}$ ); the NMDA receptor blocker APV [D(-)2-amino-5-phosphonovaleric acid; Research Biochemicals Inc.; $500 \mu \mathrm{M}$ in artificial cerebrospinal fluid (CSF)]; CPP, which is another very specific NMDA antagonist [( \pm )-3-(2-carboxypiperazin-4-yl)-propyl-1-phosphonic acid; Research Biochemicals Inc.; $1 \mathrm{mM}$ in artificial CSF]; and the AMPA receptor blocker CNQX (6-cyano-7-nitroquinoxaline-2,3-dione; Research Biochemicals Inc.; 250 $\mu_{M}$ in artificial CSF). The artificial fish CSF consisted of $124 \mathrm{~mm} \mathrm{NaCl}$, $2 \mathrm{mM} \mathrm{KCl}, 1.25 \mathrm{mM} \mathrm{KH}_{2} \mathrm{PO}_{4}, 1.1 \mathrm{mM} \mathrm{MgSO}$, $1.1 \mathrm{mM} \mathrm{CaCl}_{2}$, and 16 mM NaHCO 3 (L. Maler, personal communication; see Dye, 1988). GABA and bicuculline were injected iontophoretically (tip diameter of the electrode barrel, 5-10 $\mu \mathrm{m}$; GABA: $30-70 \mathrm{nA}$ positive DC for injection and 20-50 nA negative DC for backing current; bicuculline: ca. $100 \mathrm{nA}$ positive $\mathrm{DC}$ for injection and ca. $50 \mathrm{nA}$ negative $\mathrm{DC}$ backing current). APV , CNQX, and (as a control) CSF were pressure injected. For that purpose, a tightly fitting polyethylene tube was pushed into the open end of the electrode barrel and the other end of the tube was connected via a $\mathrm{T}$-joint to an air pressure line. By manually closing the open end of the T-joint, the pressure inside the barrel could be increased. This forced a small quantity of solution out of the tip of the electrode barrel. By visually inspecting the size of the droplet, a quantity of approximately $0.1-0.2 \mathrm{nl}$ was calculated to be ejected over a time period of $1-5 \mathrm{sec}$. Any solution of this quantity, even CSF, caused an immediate increase of the pacemaker frequency. By listening to this acceleration in pacemaker frequency, the volume of the injection could be limited additionally. In most cases, more than one drug was tested in one fish, often using one as a control for the action of the other. Each drug was tested in at least four individuals.

In the lesion experiments, first, the area of the diencephalic prepacemaker causing chirps was localized by iontophoretic stimulation with glutamate, and then, depending on the experiment, the localization of either the portion of the diencephalic prepacemaker causing gradual EOD accelerations (PPn-G) or the sublemniscal prepacemaker nucleus was determined. To test the influence of lesions of the diencephalic and sublemniscal prepacemaker nucleus, respectively, on the response to iontophoretic stimulation of the nucleus electrosensorius causing EOD accelerations (nET) or EOD decelerations ( $\mathrm{nE} \mid$ ), a second electrode was positioned in the nucleus electrosensorius by using a separate microdrive. When injecting pharmacological agents into the pacemaker nucleus, the injection electrode was positioned in the pacemaker and a stimulation electrode (advanced by a separate microdrive) was situated in either the PPn-G, the sublemniscal prepacemaker, or the nucleus electrosensorius.

The intracellular recording and labeling of neurons as well as the injection and histological reaction of choleratoxin followed a procedure described in Metzner and Heiligenberg (1991a) and Heiligenberg et al. (1991).

At the conclusion of the stimulation and lesion experiments, the fish was immersed in MS-222 and perfused with 4\% paraformaldehyde. The brain was cut frontally on a vibratome in sections of $50 \mu \mathrm{m}$ thickness, mounted, and counterstained with neutral red. The nomenclature of brain structures follows Maler et al. (1991).

\section{Results}

\section{Lesions of the medial portion of the diencephalic} prepacemaker complex

Iontophoretic injections of L-glutamate into the PPn-G (Kawasaki et al., 1988a) were used to produce gradual rises of the pacemaker frequency in order to locate the most sensitive area for subsequent lesioning. This location was determined by shifting the stimulation electrode in steps of approximately $50 \mu \mathrm{m}$ medially and rostrocaudally from the PPn-C (see Kawasaki et al., 1988a). Strongest responses to L-glutamate were produced approximately $150-200 \mu \mathrm{m}$ medial, $50 \mu \mathrm{m}$ dorsal, and $150 \mu \mathrm{m}$ rostral to the chirp-producing prepacemaker. This area, however, was located approximately $100-150 \mu \mathrm{m}$ more rostromedially than the previously described PPn-G (Heiligenberg et al., 1981; Kawasaki et al., 1988). It rather corresponded to the rostral portion of the central-posterior nucleus (CP) of the thalamus. We will include this area of the $\mathrm{CP}$ into our functional definition of the medial portion of the diencephalic prepacemaker complex ( $=$ PPn-G/CP). Most recent results from anatomical studies support this functional definition (Zupanc and Heiligenberg, 1992; G. K. H. Zupanc and M. M. Zupanc, unpublished ubservations); injections of the more sensitive neurotracers choleratoxin and Phaseolus lectin into the pacemaker nucleus revealed retrogradely labeled neurons not only in the PPn, as previously described after injections of HRP (Heiligenberg et al., 1981), but also within the adjacent CP.

After bilateral lesions of this most sensitive area for eliciting gradual EOD accelerations, the fish was no longer able to perform a complete JAR (Fig. $3 A-D$ ). The lesions eliminated any response to jamming stimuli with negative frequency differences (five fish; Fig. $3 B$ ), which usually drive the EOD frequency above the resting frequency. After the lesion, however, the EOD frequency never reached values above the resting frequency and the level of the resting frequency remained unchanged. Stimuli with positive frequency differences still lowered the pacemaker frequency after this lesion (Fig. $3 B$ ). Even after large lesions that included the laterally adjacent PPn-C (Fig. 3D), the fish still lowered its pacemaker frequency in response to positive frequency differences. The additional lesion of the PPn-C had no obvious effect.

When a jamming stimulus with negative frequency difference was presented at the onset of a stimulus sequence, the EOD frequency decreased up to $1 \mathrm{~Hz}$ below the resting frequency (Fig. $3 B$, indicated by white stars). Thus, a stimulus with a negative frequency difference that usually excited pacemaker cells and increased the EOD frequency became inhibitory after lesions of the PPn.

After lesioning the PPn, the time constants of the JAR changed as well. This is shown in Figure $3 C$, where the time constants for the JAR to stimuli with negative (i.e., EOD accelerations; arrowhead upward) and positive frequency differences (i.e., EOD decelerations; arrowhead downward) are plotted. The changes in EOD accelerations were the most apparent ones. They were significantly slower; that is, the time constant was lower after the lesion than before (paired $t$ test, $P<0.1 \%$ ). The EOD fre- 
Figure 3. Lesions of the PPn-G/CP and their effects on the JAR. The upper traces in $A$ and $B$ show the EOD frequency; the dotted line represents the resting frequency. The lower traces indicate the frequency difference $(D f)$ between jamming stimulus and EOD mimic (upward deflections, positive frequency difference; downward deflections, negative frequency difference). The dashed line represents the time span when no jamming stimulus was presented. The arrowheads in the upper traces indicate the onsets and offsets of the jamming stimulus (on and off in the lower traces). The time interval between individual traces presented here is given underneath the lower trace $(300$ sec). $A$, JAR approximately $1 \mathrm{~min}$ before the lesion. $B$, Lesion of the PPnG/CP (asterisk) eliminated the response to jamming stimuli with negative frequency differences. The EOD no longer rose above the resting frequency. The response to positive frequency differences was unchanged. Note the drop in EOD frequency during initial stimuli with negative frequency difference (indicated by the white stars). $C$, Time courses of the JAR in four fish as represented by the time constant. The time constant $\alpha$ was calculated according to the formula $0.5=\exp \left(-\alpha T_{1 / 2}\right)$, with $T_{1 / 2}$ being the interval of time over which the EOD frequency shifted halfway toward the asymptotic level. Each column represents a mean of 10 or more values. The SDs are given by the error bars above each column. Arrowheads upward, increase of EOD frequency in response to negative frequency differences (=EOD acceleration); arrowheads downward, decrease of EOD frequency due to positive frequency differences (=EOD deceleration); $R$, recovery of EOD from previous JAR with no jamming stimulus present; $R+$ arrowhead upward, recovery from previous decrease below resting frequency (i.e., "passive" acceleration). $D$, Drawing of a frontal section through the fish's diencephalon showing the bilateral lesion of the whole PPn (black areas). The lesion extended over a rostrocaudal length of approximately $250 \mu \mathrm{m}$. The asterisks indicate the centers of four individual lesions. Even after this large lesion, the fish still performed a JAK to positive frequency differences (i.e., EOD deceleration). Arrowhead downward, ventral nucleus electrosensorius. $C P$, centralposterior nucleus; $G$, glomerular nucleus; $n E$, nucleus electrosensorius; $S E$, subelectrosensorius nucleus; $T A$, nucleus tuberis anterior; $\mathrm{TeO}$, tectum opticum; $T S d$, torus semicircularis dorsalis; $V, 3$ rd ventricle.
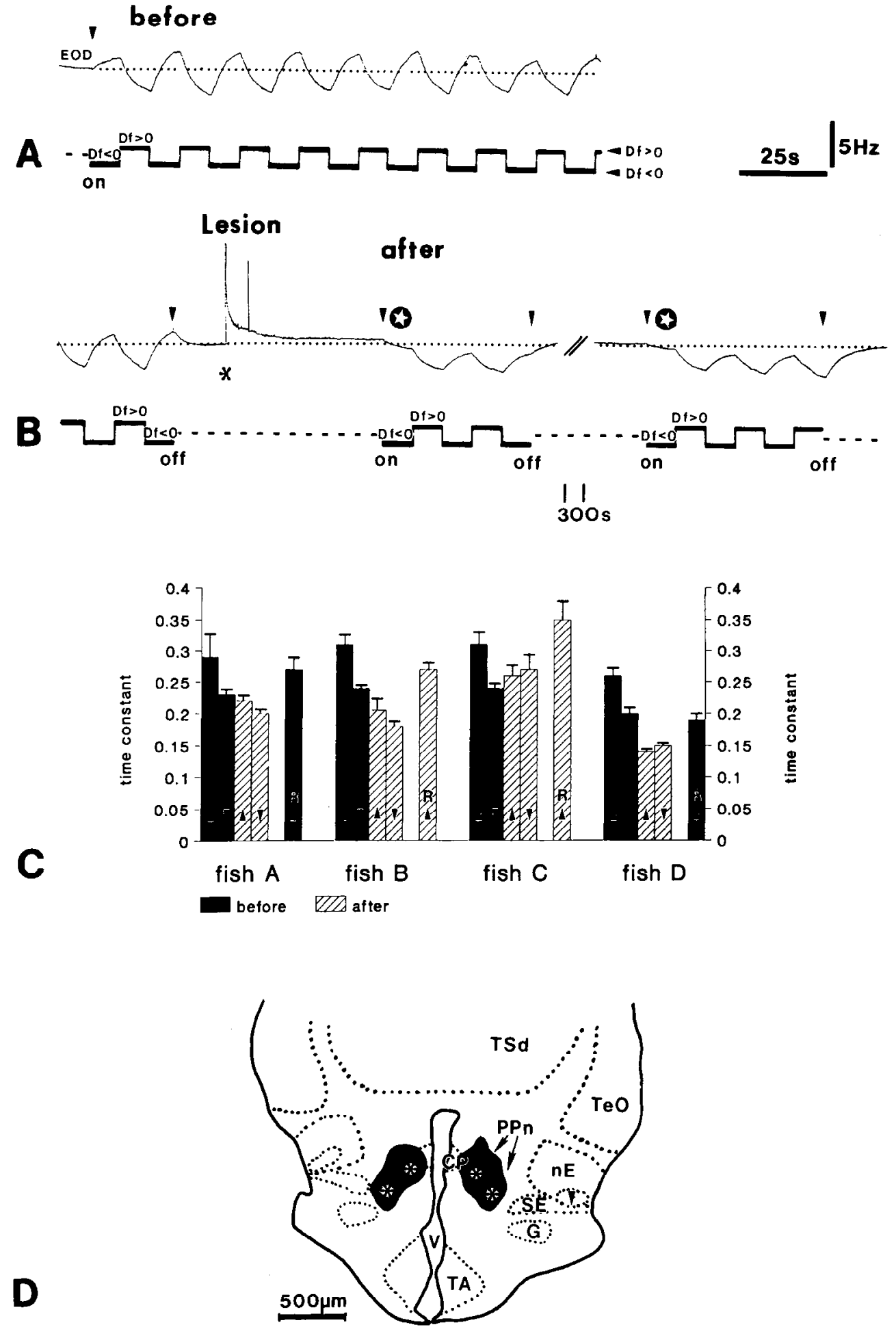

quency increased even more slowly after the lesion than it decreased before (three out of four fish; $P<0.1 \%$ ). The postlesion EOD decelerations were slower than before as well $(P<0.1 \%)$. In fact, both postlesion acceleration and deceleration were slower than the pure recovery $(P<0.1 \%)$.

Apart from the effects of these lesions on the JAR, I also tested how they affected the behavioral consequences of direct stimulation of the ipsilateral nucleus electrosensorius and of the ipsilateral SPPn (Fig. 4). Before the lesion of the PPn, L-glutamate stimulation of the dorsal nucleus electrosensorius (Fig. $4 A$, left) caused a smooth acceleration of the pacemaker while stimulation of the ventral nucleus electrosensorius (Fig. $4 B$, left) 
caused pacemaker decelerations. After the lesion, stimulation of the dorsal nucleus electrosensorius (Fig. $4 A$, right) failed to raise the EOD frequency while the response to stimulations of the ventral nucleus electrosensorius (Fig. $4 B$, right) remained intact. The response to stimulation of the SPPn (injection of GABA; see below) also remained unchanged (Fig. $4 C$ ).

Therefore, the JAR to stimuli with negative frequency differences, which involves EOD accelerations, appears to be mediated by the PPn-G/CP. In contrast, the response to jamming signals with positive frequency differences, which involves EOD decelerations, must be controlled by a neuronal pathway that bypasses the PPn-G/CP.

\section{Lesions of the sublemniscal prepacemaker nucleus}

During the course of the experiments described above, we found that the SPPn, which had recently been discovered in the related genera Sternopygus and Hypopomus (Keller et al., 1991a), also exists in Eigenmannia (Fig. 5; Keller et al., 1991b). It appeared to be a very small nucleus, however, and only a few scattered cells were backlabeled in the midbrain tegmentum after injections of Phaseolus lectin or choleratoxin into the pacemaker nucleus. They were located medioventrally to the lateral lemniscus and rostrocaudally at the level of the dorsal raphe (Fig. $5)$.

In order to determine the role of the SPPn in the control of the JAR in Eigenmannia, this area was lesioned bilaterally in a total of six fish. Before we were able to identify the SPPn pharmacologically (see below), its location in the fish's brainstem had to be determined by using its position relative to the PPn-C. The SPPn is located approximately $500-600 \mu \mathrm{m}$ caudal, 50-100 $\mu \mathrm{m}$ medial, and 100-200 $\mu \mathrm{m}$ dorsal of the center of the PPn-C.

Immediately after a unilateral lesion of the SPPn, the resting frequency dropped between 5 and $9 \mathrm{~Hz}$ below its former level and did not recover for the rest of the experiment (i.e., for at least $4 \mathrm{hr}$ ). The response to jamming signals with positive frequency differences was markedly reduced, and it was completely eliminated after bilateral lesions of the SPPn (Fig. 6A,B). EOD frequencies never decreased below the level of the new resting frequency (Fig. 6B). Nevertheless, the fish still responded to stimuli with negative frequency differences (Fig. $6 B$ ). The EOD frequency rises, however, were sometimes wobbly, and the resting frequency was often less stable than before the lesions; it varied over a range of $2-4 \mathrm{~Hz}$, corresponding to $1-5 \%$ of its absolute frequency value. The JAR disappeared completely if, in addition to lesions of the SPPn, the PPn-G was lesioned.

Usually, excitatory jamming signals with negative frequency differences caused decreases of the EOD frequency after lesions of the PPn (see Fig. $3 B$, white stars). In contrast, after lesions of the SPPn, the onset of a stimulus with a positive frequency difference never caused the EOD frequency to increase (Fig. $6 B$, indicated by the white star).

An intact fish shifts its EOD frequency faster upward than downward (Figs. $3 C, 6 C$, solid columns; see also Bullock et al., 1972; Bastian and Yuthas, 1984). After lesions of the SPPn, however, this relation was inverted; decelerations of the pacemaker were faster than accelerations (Fig. 6C) (paired $t$ test, $P$ $<0.1 \%$ ). However, much as with lesions of the PPn, the increase in the EOD frequency was generally slower than before $(P<$ $0.1 \%)$.

Figure 7 shows the effects of lesions of the SPPn on the behavioral responses to stimulation of both the ipsilateral nucleus electrosensorius and of the PPn-G/CP. After the lesion, the

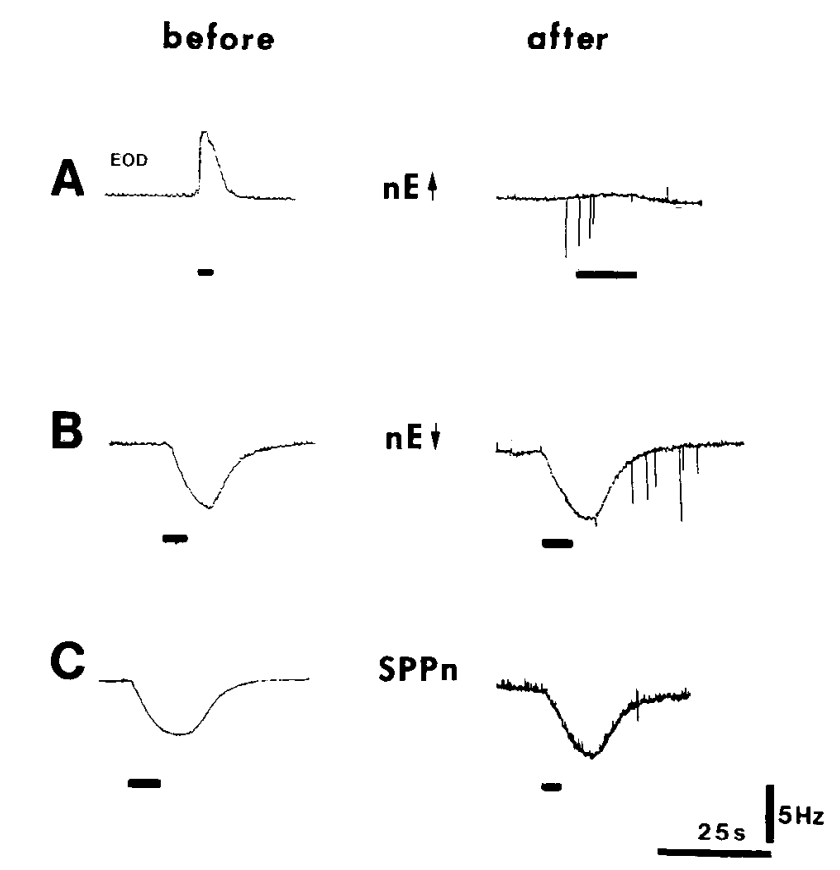

Figure 4. EOD frequency changes in response to stimulation of the dorsal nucleus electrosensorius $(n E \eta)$, ventral nucleus electrosensorius $(n E \downarrow)$, and SPPn before and after lesions of the ipsilateral PPn-G/CP. The stimulus duration is indicated by the bars beneath the traces. $A$, Lesion of the PPn eliminated the response to L-glutamate (100 mM) in the dorsal nucleus electrosensorius. $B$, The response to stimulation of the ventral nucleus electrosensorius with L-glutamate was not affected. $C$, Similarly, the effect of stimulation of the SPPn with GABA (500 mM; see Fig. 7) did not change after the lesion. The irregular spikes in the right traces of $A$ and $B$ (after the lesion) represent abrupt frequency modulations ( $=$ chirps) that were produced as a result of lesioning parts of the PPn-C.

frequency decrease in response to glutamate stimulation of the ventral nucleus electrosensorius was eliminated (Fig. $7 B$ ) while the stimulation of the dorsal nucleus electrosensorius (Fig. 7A) and of the PPn-G/CP (Fig. 7C) still elicited gradual EOD accelerations. Therefore, the control of the JAR to stimuli with positive frequency differences appears to be mediated by the SPPn.

\section{GABAergic input to the sublemniscal prepacemaker nucleus}

In contrast to our experience in other gymnotiform fish (Sternopygus, Keller et al., 1991a; Apteronotus, W. Heiligenberg and W. Metzner, unpublished observations; and Hypopomus, Heiligenberg, personal communication), the SPPn in Eigenmannia could not be stimulated by electrophoretic injections of L-glutamate (see below). However, electrical pulses between 1 and $15 \mu \mathrm{A}$ (ca. 1 msec duration at about $200 \mathrm{~Hz}$; eight trials in three fish) caused slight increases of the EOD frequency of up to 3 $\mathrm{Hz}$ (Fig. 8A, left). The performance of the JAR was not altered significantly by the electrical stimulation; the magnitude of the response to signals with positive and negative frequency differences, respectively, remained unchanged (Fig. $8 A$, right).

Electrophoretic injections of the inhibitory transmitter GABA into the SPPn reduced the EOD frequency by as much as $7 \mathrm{~Hz}$ (Fig. $8 B$, left). The resting frequency returned then to its former level within a few seconds after the end of the injection. The frequency changes due to GABA injection into the SPPn resembled very much those observed during injection of L-glutamate into the ventral nucleus electrosensorius (see Fig. $8 D$, nE $\downarrow$ ). During the JAR, GABA injections into the SPPn shifted the baseline 


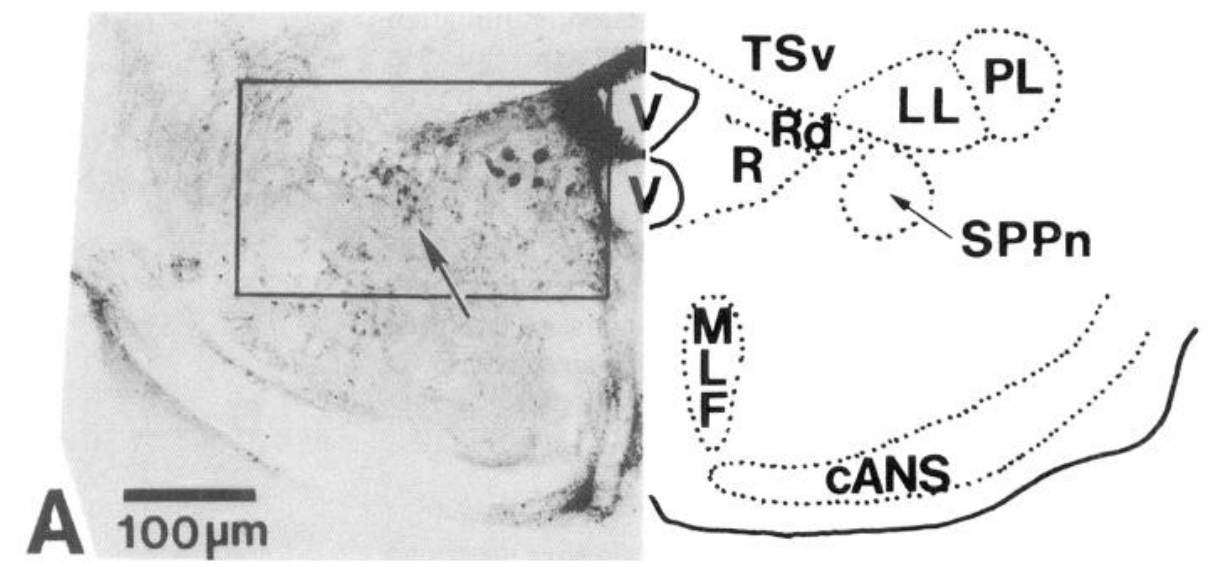

Figure 5. Photomicrograph of a frontal section through the brainstem tegmentum of Eigenmannia showing the SPPn. A few scattered cells were retrogradely labeled after injection of choleratoxin into the medullary pacemaker nucleus (left arrow). The rostrocaudal level of this frontal section corresponds to the level of section 13 in the brain atlas by Maler et al. (1991). $A$, Overview. $B$, Detail as indicated by the boxed region in $A$. cANS, commissura ansulata; $L L$, lateral lemniscus; $M L F$, medial longitudinal fasciculus; $P L$, paralemniscal nucleus; $R$, red nucleus; $R d$, dorsal raphe; $T S v$, torus semicircularis ventralis; $V$, fourth ventricle.

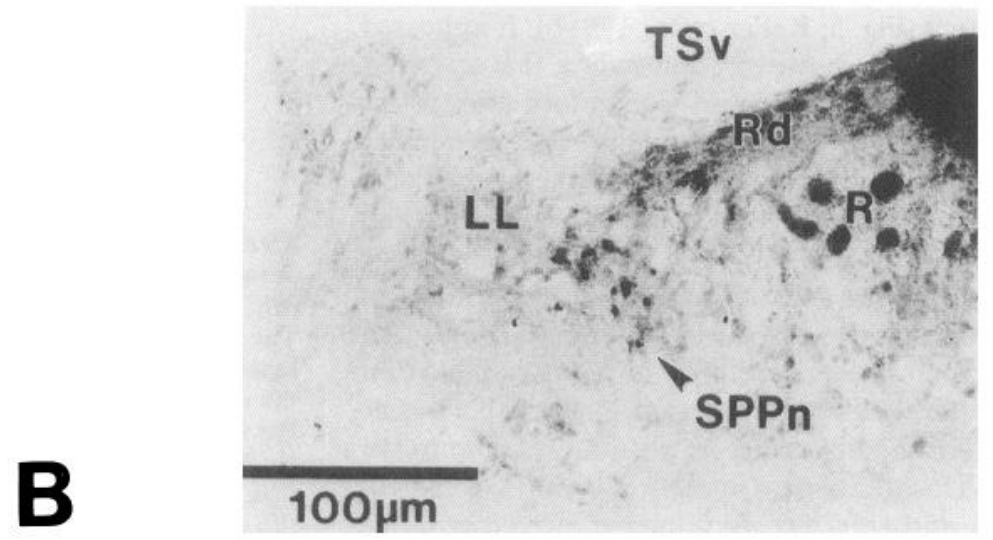

up to $7 \mathrm{~Hz}$ below the original resting frequency (Fig. $8 B$, right). The magnitude of the response to stimuli with positive frequency difference was not notably affected. The best response to GABA was found in a very restricted area of only approximately $100-200 \mu \mathrm{m}$ in diameter that corresponds to the center of the SPPn. The location of the stimulation sites was verified by injections of Alcian blue or choleratoxin. These results were confirmed in numerous trials in a total of six fish. Injections of $\mathrm{NaCl}(165 \mathrm{~mm}, \mathrm{pH} 3.2)$ as a control had no effect on the resting frequency or on the JAR.

The negative results of previous injections of L-glutamate into the SPPn were confirmed by injecting glutamate at the same sites where GABA injections elicited strongest effects. No response to glutamate was observed.

To confirm the GABAergic input of the SPPn, bicuculline methiodide, a GABA antagonist, was injected electrophoretically. Bicuculline induced a slight increase of the resting frequency by as much as $4 \mathrm{~Hz}$ above its former level (Fig. $8 C$, left). The bicuculline reaction was very slow; the frequency increase occurred over a period of several hundred seconds during continuous bicuculline injection, and recovery required up to 3 min. During the JAR, bicuculline injection caused a slight reduction in the response to stimuli with positive frequency difference and a rise of the baseline frequency (Fig. $8 C$, right).

In another set of experiments, the ventral nucleus electrosensorius was stimulated with glutamate during simultaneous bicuculline injection into the ipsilateral SPPn. The frequency decrease in response to the stimulation of the ventral nucleus electrosensorius ( $\mathrm{nE}$, Fig. $8 D$ ) could be blocked by injection of bicuculline into the SPPn (Fig. 8D). It took several minutes for the stimulation of the ventral nucleus electrosensorius to reach its former magnitude.

These experiments suggest that the SPPn receives tonic inhibitory, GABAergic input from the ventral nucleus electrosensorius. Whether the ventral nucleus electrosensorius is directly connected with the SPPn or whether this connection is mediated via yet unknown links remains to be determined by anatomical experiments.

Control of the pacemaker nucleus by the diencephalic and the sublemniscal prepacemaker nuclei

The medullary pacemaker nucleus in Eigenmannia contains intrinsic pacemaker neurons and relay neurons that innervate the electromotoneurons in the spinal cord. Pacemaker and relay cells are coupled electrotonically, while the inputs from the PPn and from the SPPn are mediated by chemical synapses (Bennett, 1971; Dye and Meyer, 1986; Szabo et al., 1989). Immunohistochemical as well as pharmacological studies did not reveal any evidence for the presence of inhibitory transmitters in the pacemaker nucleus of Eigenmannia (Kawasaki and Heiligenberg, 1990; G. Kennedy, personal communication).

In order to test the pharmacological nature of pacemaker afferents, the AMPA antagonist CNQX and the NMDA antagonist APV were injected into the pacemaker nucleus. Their effects on the JAR and on the efficacy of the nucleus electrosensorius and of the PPn and the SPPn were tested (see also Dye et al., 1989).

Injection of CNQX into the pacemaker drastically reduced 

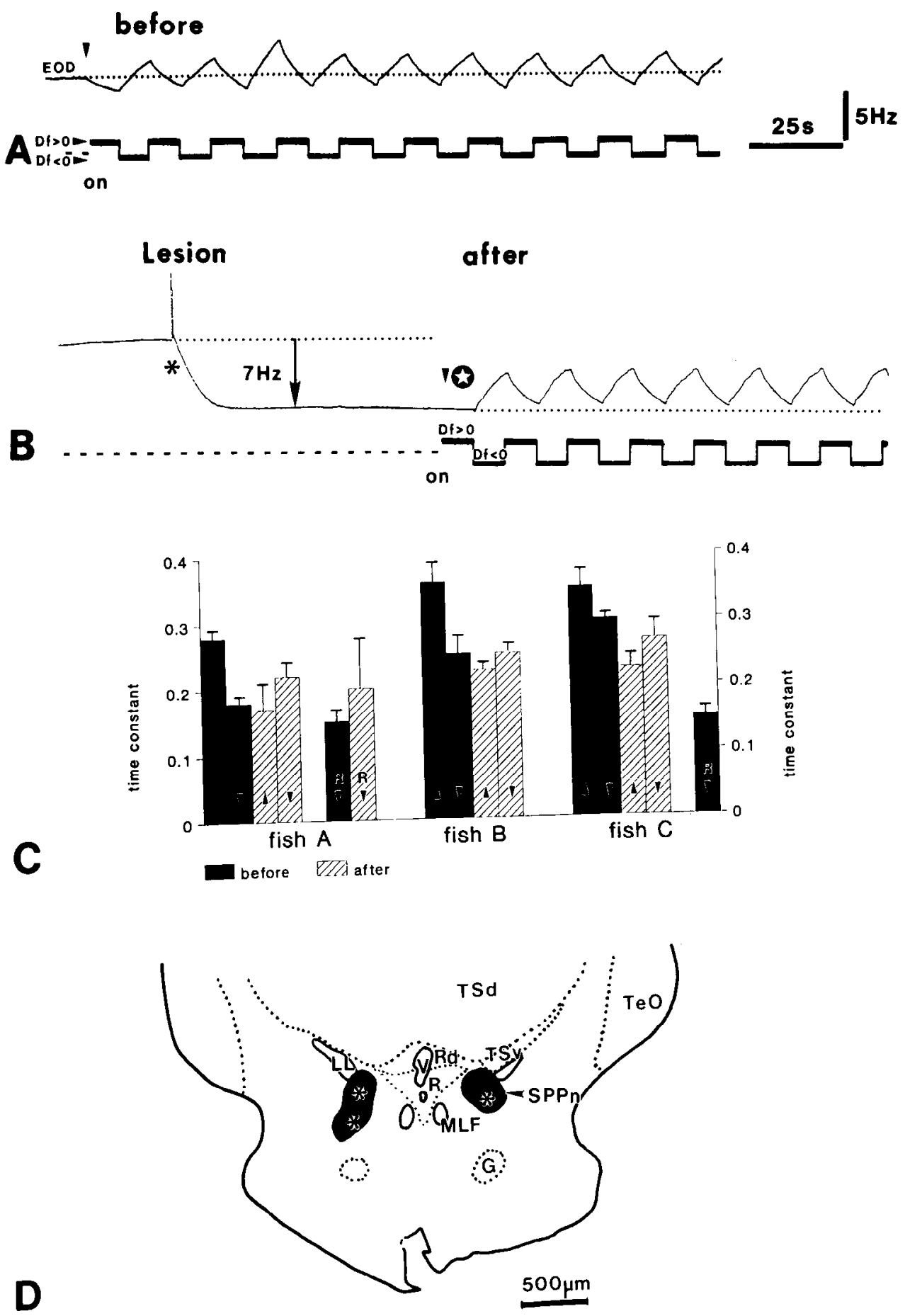

Figure 6. Lesion of the SPPn and its effects on the JAR. The presentation of data follows the convention shown in Figure 3. $A$, JAR approximately $5 \mathrm{~min}$ before the lesion of the SPPn. $B$, The lesion of the SPPn (asterisk) caused a decrease of the resting frequency and eliminated the JAR to positive frequency differences. The fish was no longer able to lower its EOD frequency below the new resting frequency. However, the fish still avoided jamming signals with negative frequency difference by increasing its EOD frequency. Note that, in contrast to the situation after lesions of the PPn, there was no response to the onset of a positive frequency difference (white star). $C$, Time constants for EOD frequency changes during JAR before and after lesion of the SPPn. See Figure $3 C$ for explanation of symbols. $R+$ arrowhead downward, recovery from previous increase above resting frequency (i.e.. "passive" deceleration). $D$, Drawing of a frontal section through the level of the brainstem tegmentum showing the lesion of the SPPn (black). The lesion extended over a rostrocaudal distance of approximately $200 \mu \mathrm{m}$. Compare with Figure 5 for the location of the SPPn. $G$, glomerular nucleus; $L L$, lateral lemniscus; $M L F$, medial longitudinal fasciculus; $R$, red nucleus; $R d$, nucleus raphe dorsalis; $T e O$, tectum opticum; $T S d$, torus semicircularis dorsalis; $T S v$, torus semicircularis ventralis; $V$, fourth ventricle. the response to jamming signals with negative frequency differences, that is, EOD accelerations (Fig. 9A-C). The JAR very much resembled that obtained after lesion of the PPn (see Fig. 3 ), and the baseline frequency of the JAR decreased (Fig. 9B). The levels of the resting frequency and the JAR to stimuli with positive frequency differences, that is, the EOD deceleration, were not affected (Fig. 9B). The JAR recovered after several hundred seconds (Fig. 9C). A control injection of artificial CSF into the pacemaker nucleus had no significant effect on the performance of the JAR (Fig. 10).

CNQX also reduced the response to glutamate stimulation of the medial portion of the PPn, which causes gradual EOD ac- celerations, of its lateral, chirp-producing portion, and of the dorsal nucleus electrosensorius. The magnitude of responses to stimulation of the PPn-G/CP (Fig. 11A) and of the dorsal nucleus electrosensorius (Fig. $11 B$, nE $\rceil$ ) decreased after 10-20 sec and slowly recovered after several hundred seconds. In accordance with earlier findings by Dye et al. (1989), CNQX also blocked the input from the PPn-C (not shown).

The responses to glutamate stimulation of the ventral nucleus electrosensorius (Fig. $11 D, \mathrm{nE} \mid$ ) and to GABA stimulation of the SPPn (Fig. 11C) were not affected. Control injections of artificial CSF also had no effect, which is shown in Figure $11 \mathrm{E}$ for the stimulation of the PPn-G/CP. 


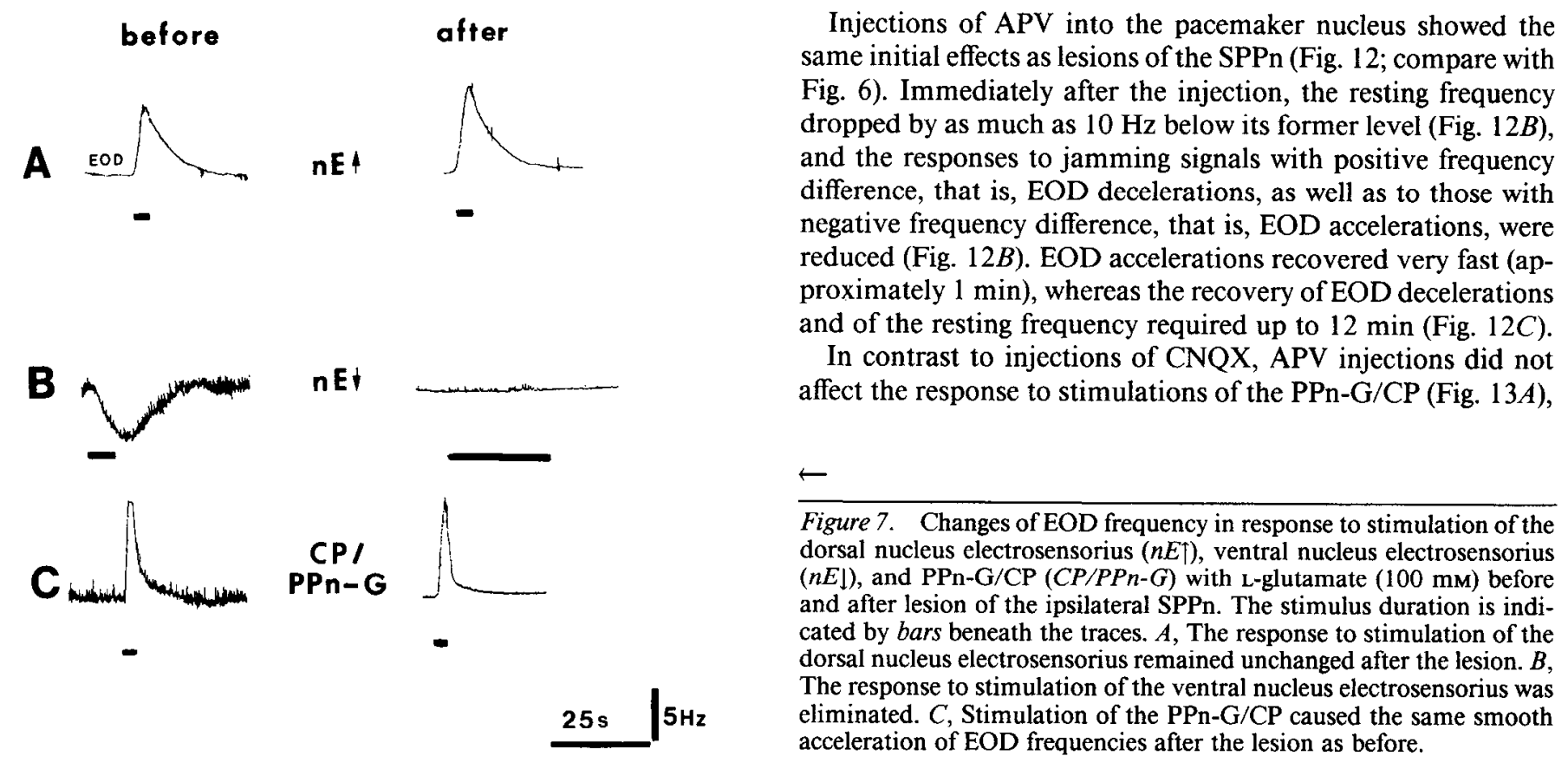

Injections of APV into the pacemaker nucleus showed the 6) inial effects as lesions of the SPPn (Fig. 12; compare with dropped by as much as $10 \mathrm{~Hz}$ below its former level (Fig. 12B), and the responses to jamming signals with positive frequency difference, that is, EOD dccelcrations, as well as to those with negative frequency difference, that is, EOD accelerations, were reduced (Fig. 12B). EOD accelerations recovered very fast (approximately $1 \mathrm{~min}$ ), whereas the recovery of EOD decelerations and of the resting frequency required up to $12 \mathrm{~min}$ (Fig. 12C)

In contrast to injections of CNQX, APV injections did not

B ELECTRICAL

A

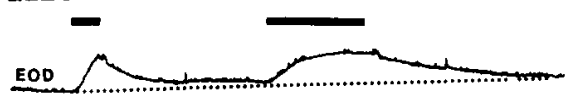

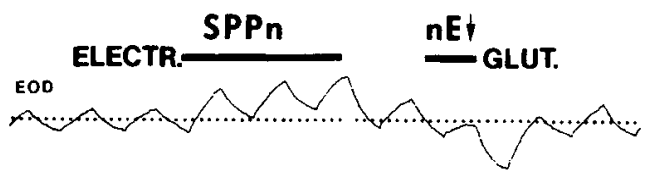

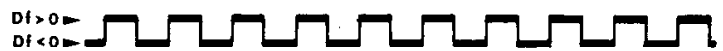

GABA

B

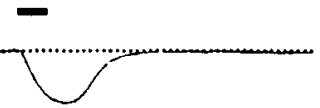

GABA

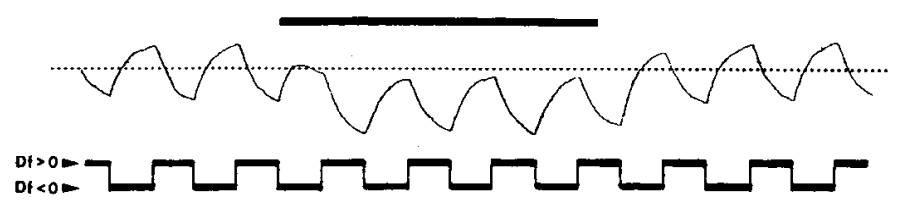

BICUCULLWE

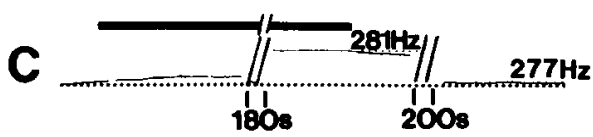

BICUC.

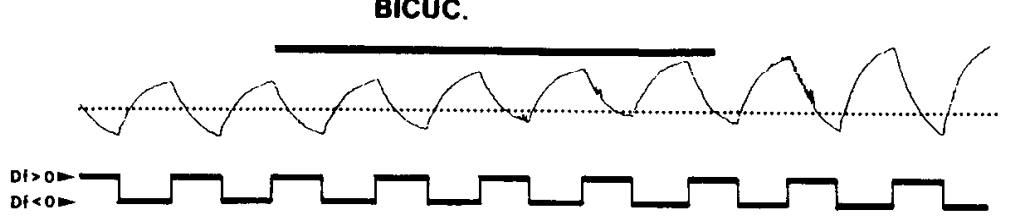

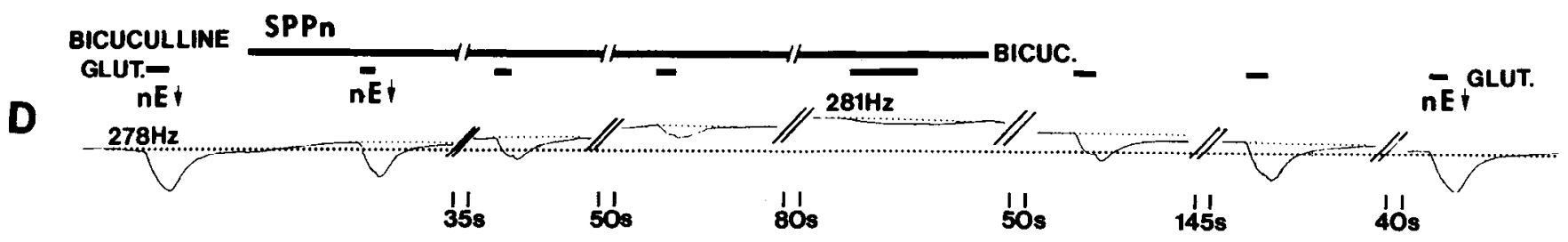

Figure 8. Effects of various stimulations of the SPPn. Left side in $A-C$, Effect upon the resting frequency; right side in $A-C$, effect upon the JAR. $A$, Electrical stimulation (negative DC pulses of $1-15 \mu \mathrm{A}$, approximately $1 \mathrm{msec}$ duration at about $200 \mathrm{~Hz}$ repetition rate) caused an increase in the resting frequency $(l e f t)$ and an increase in the baseline frequency during JAR performance (right). Note that glutamate stimulation of the ventral nucleus electrosensorius $(n E[)$ also lowered the baseline frequency. The magnitude of the JAR was not affected notably. $B$, Stimulation with GABA $(500 \mathrm{~mm})$ decreased the resting frequency (left) and also lowered the baseline frequency during the JAR (right). $C$, Bicuculline methiodide ( 2 mM) increased the resting frequency (left) and also slightly reduced the response to stimuli with positive frequency differences (right). $D$, Injection of bicuculline methiodide into the SPPn (duration indicated by the upper bar) and simultaneous repetitive stimulation of the ipsilateral ventral nucleus electrosensorius $(n E l)$ with L-glutamate $(500 \mathrm{~mm}$; indicated by the lower bars). Bicuculline blocked the EOD frequency decrease in response to stimulation of the ventral nucleus electrosensorius. 


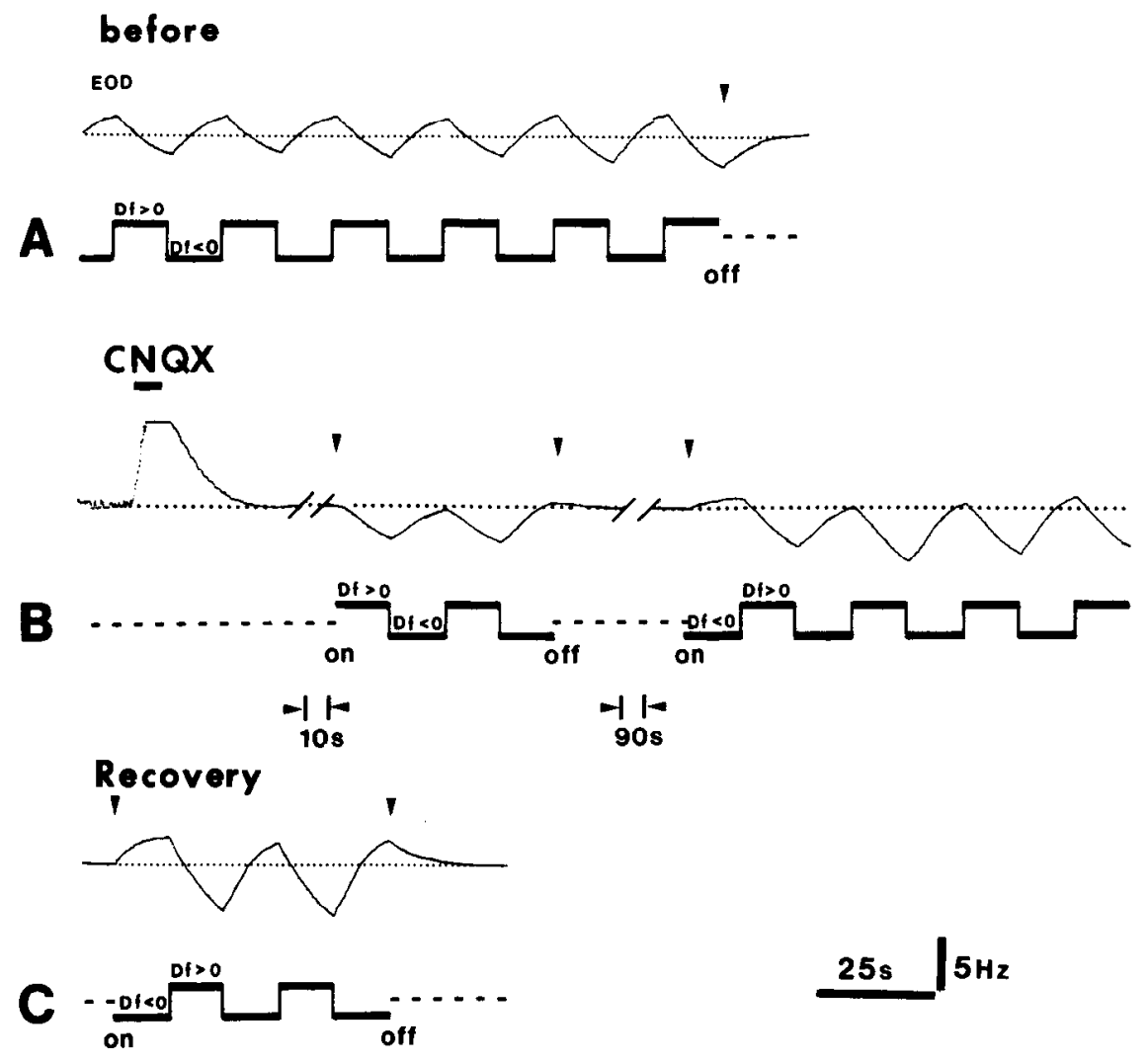

Figure 9. Injection of the AMPA antagonist CNQX (500 $\mathrm{mm}$ ) into the pacemaker nucleus and its effect on the JAR. The presentation of data follows the convention of Figure 3. $A$, JAR 100 sec before the injection. $B$, Injection of CNQX (duration indicated by bar above trace) caused the EOD frequency to remain below the resting frequency. $C$, The JAR recovered approximately 14 min after the injection. of the PPn-C (not shown), and of the dorsal nucleus electrosensorius (Fig. 13B, nE ), whereas they reduced the response to GABA stimulation of the SPPn (Fig. 13C) and to glutamate stimulation of the ventral nucleus electrosensorius (Fig. 13D, $\mathrm{nE} \downarrow$ ). The responses recovered after several minutes. Injections of artificial CSF as a control had no effect. This is demonstrated in Figure $13 E$ for the stimulation of the SPPn.

The injection of CPP, another very selective NMDA antagonist, into the pacemaker had the same effects as injections of APV.

These results indicate that the inputs from the PPn-G/CP and from the SPPn to the medullary pacemaker nucleus are medi- ated by different types of receptors. The afferents from the PPnG/CP utilize AMPA receptors and can therefore be selectively blocked by CNQX, while the afferents from the SPPn utilize NMDA-type reccptors, which can be blocked sclectively by APV.

\section{Connection between the dorsal torus semicircularis and the} nucleus electrosensorius

Jamming signals with negative frequency differences causes excitation in the dorsal nucleus electrosensorius, and this activation increases the pacemaker frequency, while signals with positive frequency differences excite units in the ventral nucleus electrosensorius, which in turn decrease the pacemaker fre-

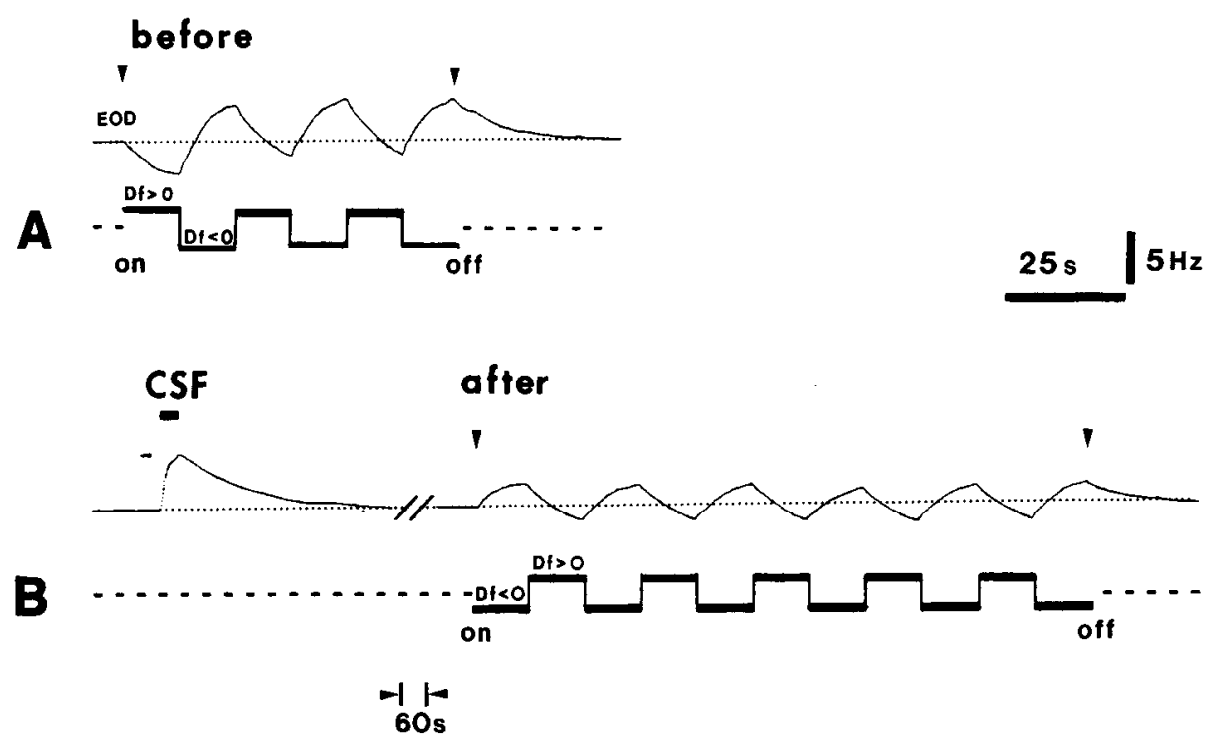

Figure 10. Control injection of artificial fish CSF into the pacemaker nucleus. The presentation of data follows the convention of Figure 3. $A$, JAR approximately $120 \mathrm{sec}$ before the injection. $B$, Injection of CSF (duration indicated by bar above trace) slightly reduced the magnitude of the JAR but did not affect the level of the resting frequency. 


\section{before CNQX after}

Figure 11. Effects of CNQX injections into the pacemaker nucleus on the response to stimulations of brain structures that mediate EOD accelerations $(A, B)$ and EOD decelerations $(C, D)$ of the JAR, respectively. The duration of the CNQX injection is marked by the bar above each trace; the duration of each stimulation is indicated by bars beneath each trace. The vertical bars to the right represent a change in the EOD frequency of $5 \mathrm{~Hz}$. The level of the resting frequency is illustrated by the broken lines. The traces represent data from three different fish $(A+E, B+D$, and $C$ were obtained from one fish each). The time intervals between individual stimulations are given by the numbers beneath each trace. $A, \mathrm{CNQX}$ injections into the pacemaker drastically reduced the gradual EOD acceleration elicited by glutamate stimulation $(100 \mathrm{~mm})$ of the PPn-G/CP. B, Similarly, the response to stimulation of the dorsal nucleus electrosensorius $(n E \uparrow)$ with glutamate was reduced. $C$, The decrease of EOD frequencies due to the stimulation of the SPPn with GABA $(500$ mM) was not affected. $D$, The pacemaker deceleration in response to glulamate stimulation of the ventral nucleus electrosensorius $(n E])$ was not notably affected as well. $E$, The control injection of artificial CSF into the pacemaker nucleus had no effect on the response to glutamate stimulation of the PPn-G/CP.

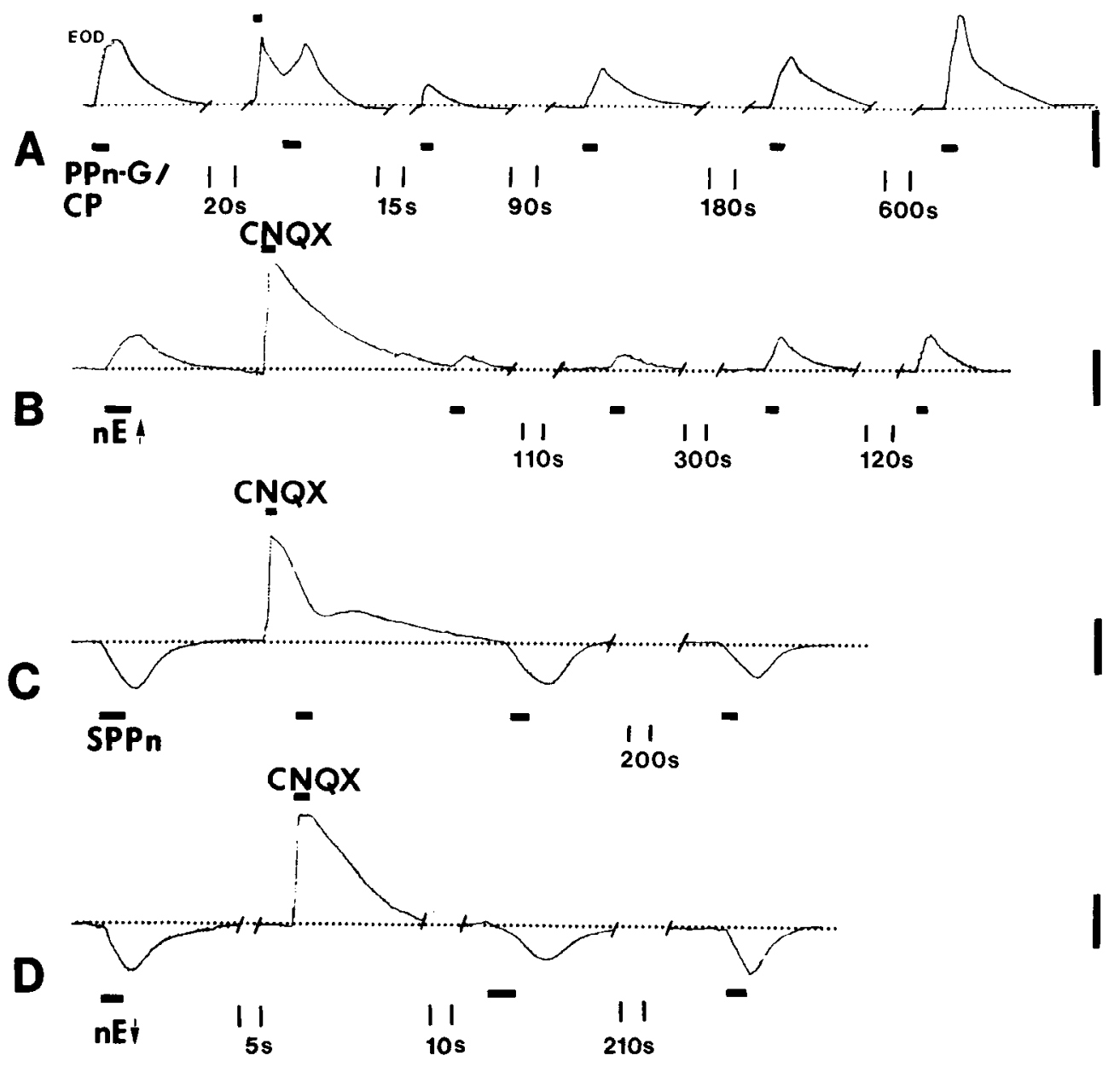

\section{CONTROL}

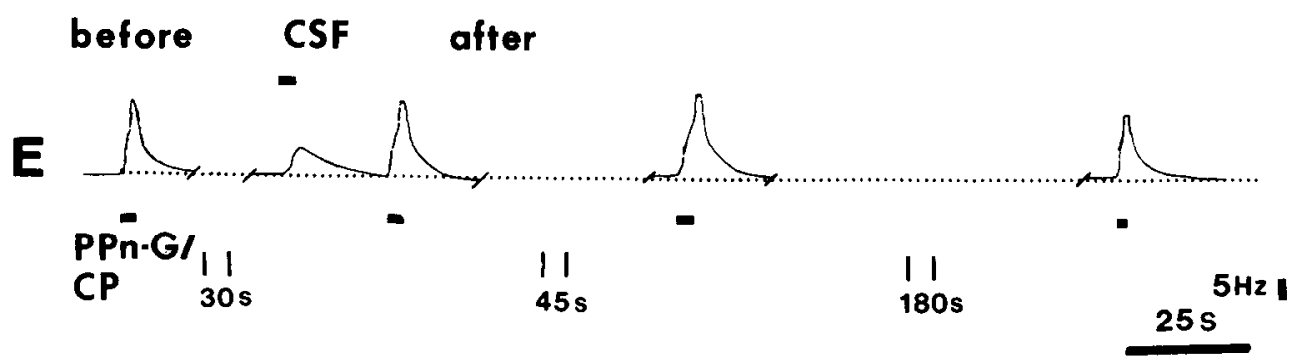

quency (Keller, 1988, 1989; Keller and Heiligenberg, 1989; see Figs. 1,2). How is this sign selectivity of neuronal responses in the dorsal and in the ventral nucleus electrosensorius, respectively, achieved? Since sign-selective neurons are already found in the torus semicircularis dorsalis (Heiligenberg and Rose, 1985, 1986; Rose and Heiligenberg, 1985a), one might assume that the dorsal nucleus electrosensorius (see Figs. 1, 2, nE $)$ ) should receive input from toral neurons excited mainly by negative frequency differences, whereas the ventral nucleus electrosensorius (Figs. 1, 2, nEl) is innervated by toral neurons excited primarily by positive frequency differences. Intracellular recording and labeling techniques can help clarify this question. By using HRP and Lucifer yellow as intracellular tracers, Rose and Heiligenberg (1985a) were able to demonstrate that these neurons were predominantly located in the deeper laminae of the dorsal torus, mainly in lamina 8c. Unfortunately, axonal projections to the diencephalon could not be found with the methods used in their study.

In the present study, by using biocytin as an intracellular marker, a sign-selective cell was characterized physiologically 


\section{before}
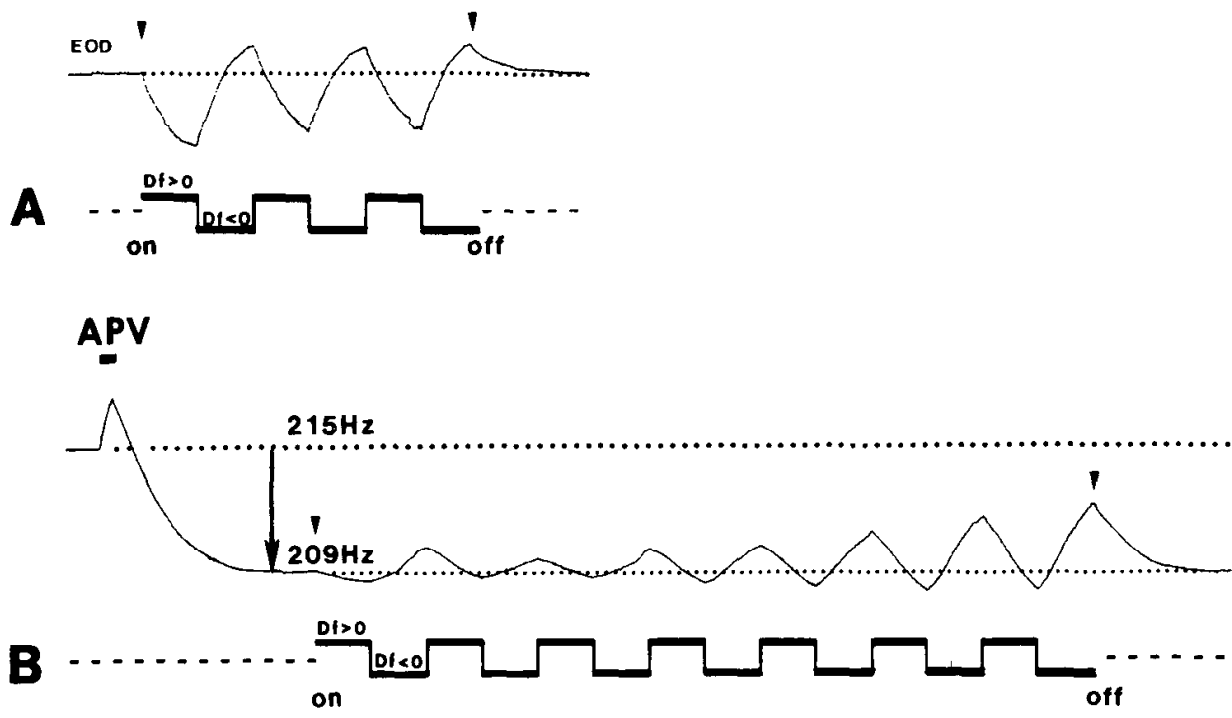

\section{Recovery}
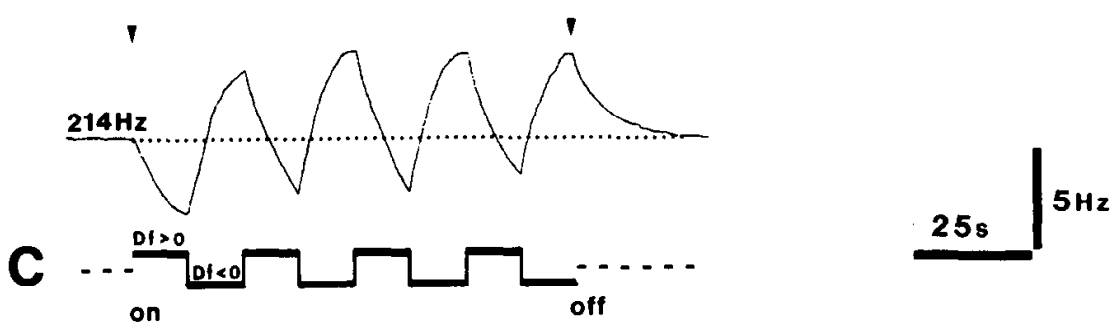

Figure 12. Injection of the NMDA antagonist $A P V$ into the pacemaker nucleus and its effect on the JAR. Presentation of data is as in Figures 3 and 9. $A$, JAR $60 \mathrm{sec}$ before the injection. $B$, Injection of APV (duration is indicated by the bar above the upper trace) caused a decrease of the resting frequency (dotted lines) from $215 \mathrm{~Hz}$ to $209 \mathrm{~Hz}$ and initially reduced the EOD acceleration as well as deceleration. EOD acceleration recovered much earlier than the deceleration (after approximately 1 min). Compare also with the control experiment shown in Figure 10. $C$, Recovery of EOD decelerations occurred after approximately $12 \mathrm{~min}$. and subsequently labeled (Fig. 14). Sign-selective neurons in the dorsal torus semicircularis are seldom found, and the recordings are rarely sufficiently stable to identify the cell and to inject a sufficient amount of tracer (at least $10 \mathrm{~min}$ ). Therefore, this is the only successfully identified and sufficiently labeled neuron so far.

This neuron responded preferably to positive frequency differences (Fig. 14A,B). If the jamming stimulus and the EOD mimic were added electronically and presented through the same pair of electrodes (situation of "identical geometry"), no phase information but only information about the amplitude of the mixed signal was contained. In this situation, the neuron was excited by the decrease in amplitude (I-unit) (Fig. 14C). The neuron was located in the ventral portion of lamina 8c (Fig. $14 D$ ) and belonged to the pyramidal cell type (Carr and Maler, 1985). It sent a prominent axon to the optic tectum. Just ventrally of the torus, a thin collateral was found heading rostrally to the ipsilateral nucleus electrosensorius. It passed through the dorsal nucleus electrosensorius without making any obvious contact on cells, and no axonal swellings could be seen within the dorsal nucleus electrosensorius (Fig. 14D, inset, nE $\uparrow$ ). However, within the ventral nucleus electrosensorius $(\mathrm{nE} \downarrow)$ a collateral turned off and terminated at the dorsal border of the ventral nucleus electrosensorius. The axon headed farther rostrally and could be followed halfway through the postoptic commissure before it was lost. From extracellular tracer injections in the dorsal torus semicircularis and in the nucleus electrosensorius
(Keller et al., 1990; Heiligenberg and Metzner, unpublished observations) and from intracellular labeling of other, non-signselective toral neurons that projected to the nucleus electrosensorius (Metzner and Heiligenberg, 1991), it is known that efferent fibers from the dorsal torus cross over through the postoptic commissure to the contralateral nucleus electrosensorius. The finding of this toral neuron therefore supports the hypothesis that the ventral nucleus electrosensorius should receive input from toral neurons responding preferably to positive frequency differences (see Figs. 1, 2).

\section{Discussion}

Separate motor pathways control EOD accelerations and EOD decelerations of the $J A R$

The current understanding of the motor control of the JAR is summarized in Figure 2 (compare with Fig. 1). Two separate pathways diverge from sign-selective cells of the dorsal torus semicircularis (TSd). The pathway controlling gradual EOD accelerations involves those toral neurons that respond selectively to negative frequency differences, the dorsal nucleus electrosensorius (nE $\uparrow$ ), the PPn-G/CP, and the primarily AMPA-mediated input to the pacemaker (Pn). This is supported by the observation that bilateral lesions of the PPn-G/CP eliminated the response to jamming signals with negative frequency differences, that is, EOD accelerations, and left the response to stimuli with positive frequency differences, that is, EOD decelerations, intact. Moreover, injections of CNQX, which selectively blocks 


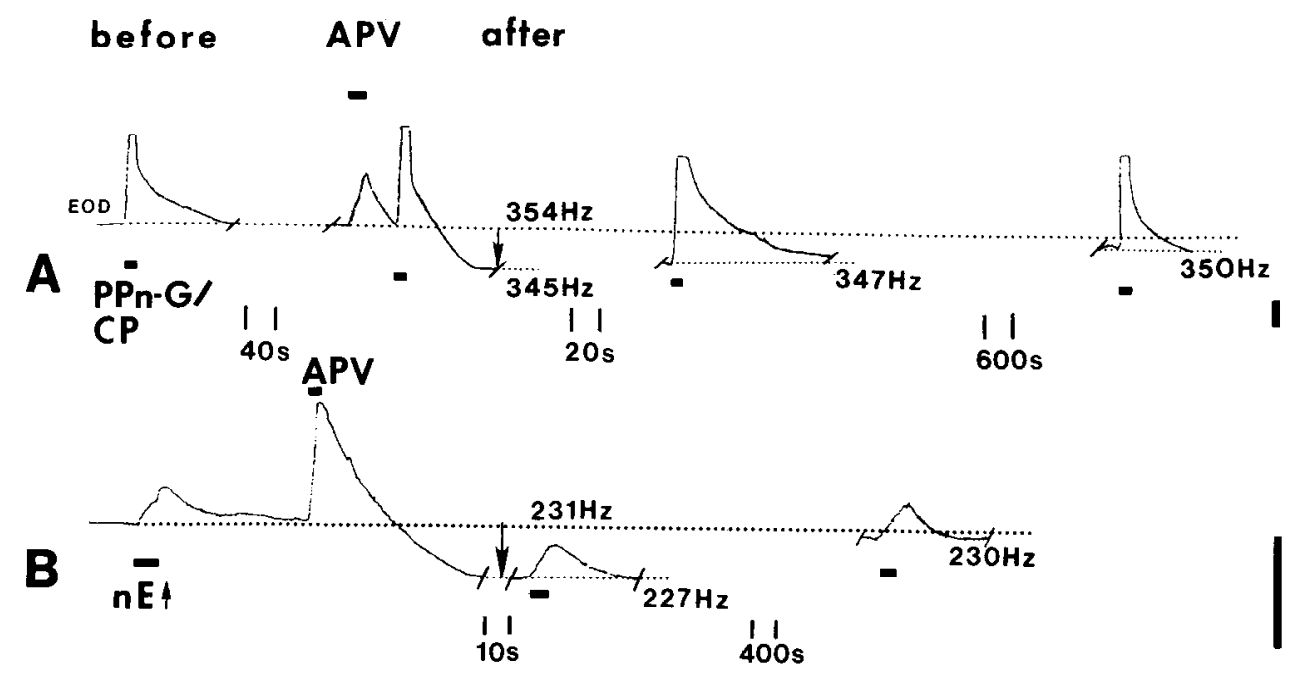

Figure 13. Effects of APV injections into the pacemaker nucleus on the response to stimulations of brain structures that mediate EOD accelerations $(A, B)$ and EOD decelerations $(C, D)$ of the JAR, respectively. The presentation of data follows the convention of Figure 11. The traces show data obtained from three different fish $(A, B+C+E, D)$. In all cases the resting frequency dropped immediately after the injection of APV and returned to its former level over a period of a few hundred seconds (down. ward arrows in $\mathrm{A}-\mathrm{D}) . A$, APV injcctions into the pacemaker nucleus did not affect the gradual rises of EOD frequencies in response to glutamate stimulation (100 mM) of the PPn-G/CP. B, It also did not influence the response to stimulation of the dorsal nucleus electrosensorius $(n E \mid)$. $C$, The deceleration of pacemaker frequencies caused by stimulation of the SPPn with GABA (500 mM) was reduced after APV injections and recovered after a few hundred seconds. $D$, Similarly, the decline in EOD frequency induced by glutamate stimulation of the ventral nucleus electrosensorius $(n E \downarrow)$ was diminished after the injection of APV. $E$, Control injections of CSF had no effect on the response to GABA stimulations of the

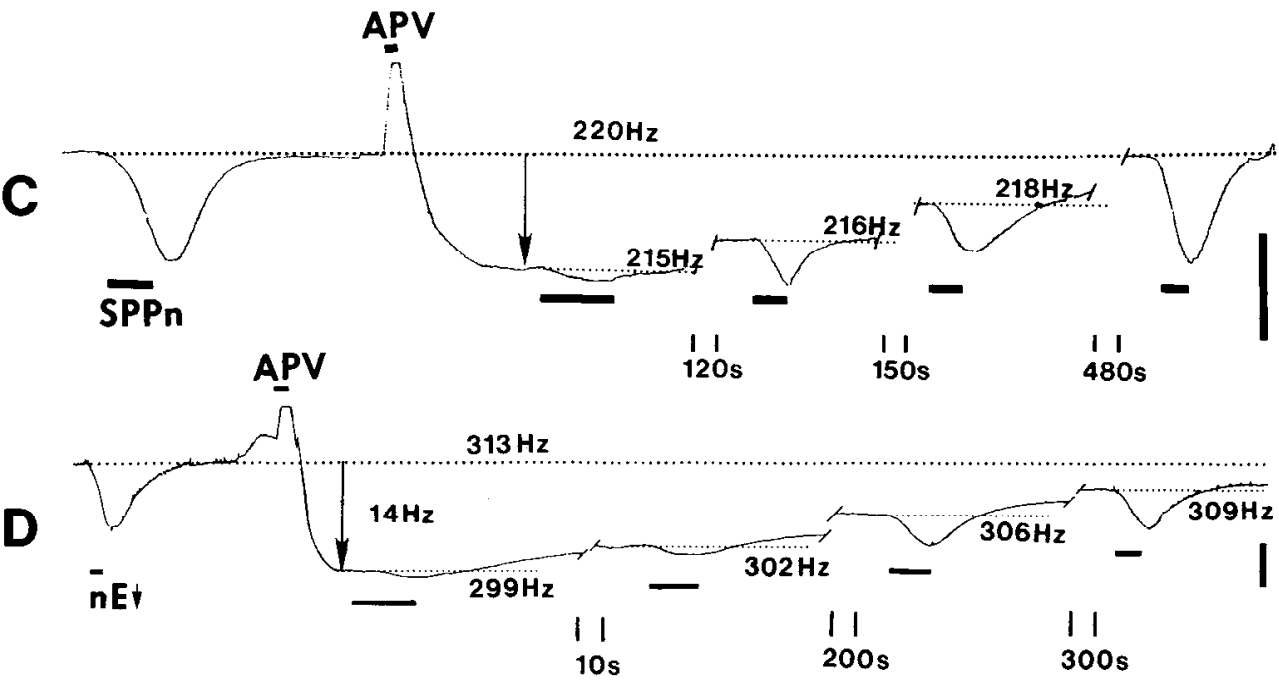

CONTROL

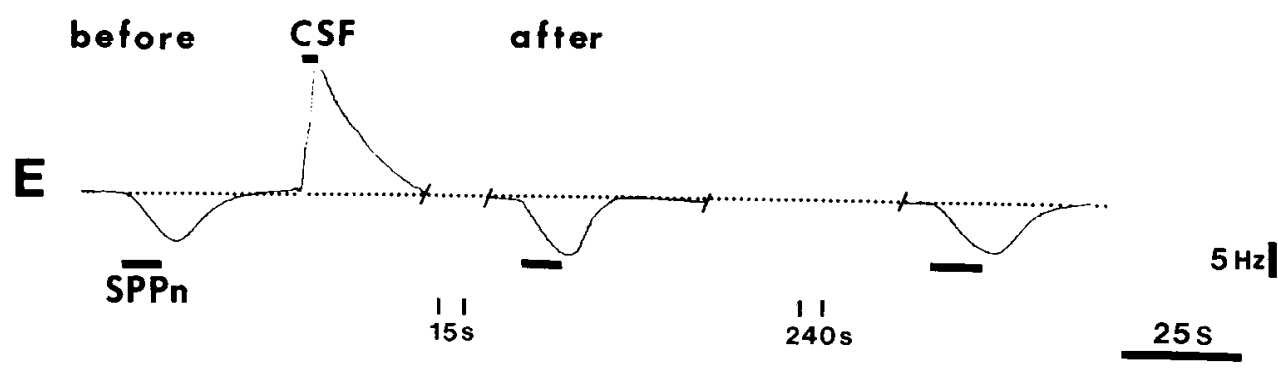
SPPn.

AMPA-type receptors, into the pacemaker nucleus ( $\mathrm{Pn}$ ), had results much like lesions of the PPn-G/CP. The fast time courses of EOD accelerations in intact fish (Bullock et al., 1972; Bastian and Yuthas, 1984; see also Figs. 3, 6) may in part reflect the fast synaptic transmission provided by AMPA-type receptors. However, the response to negative frequency differences (i.e., EOD accelerations) was initially also reduced after injection of APV, an NMDA antagonist, into the pacemaker (Fig. 12B). Although this effect was not too different from the effect of the control injection of CSF (Fig. 10), it may suggest that the input from the PPn-G/CP is at least partially also mediated by NMDAtype receptors.
The pathway that controls EOD decelerations starts with neurons in the dorsal torus semicircularis that are selective for positive frequency differences. It subsequently involves the ventral nucleus electrosensorius ( $\mathrm{n} E \downarrow$ ), which in turn lowers the tonic activity of the SPPn via its GABAergic input. The diminished activity of the SPPn finally reduces the NMDA-mediated input to the pacemaker (Pn). GABAergic transmission and NMDA transmitter types represent rather slow synaptic mechanisms. This might cause EOD decelerations to be generally much slower than EOD accelerations. This pathway is suggested by the fact that bilateral lesions of the SPPn eliminated the response to stimuli with positive frequency differences, that is, EOD decel- 


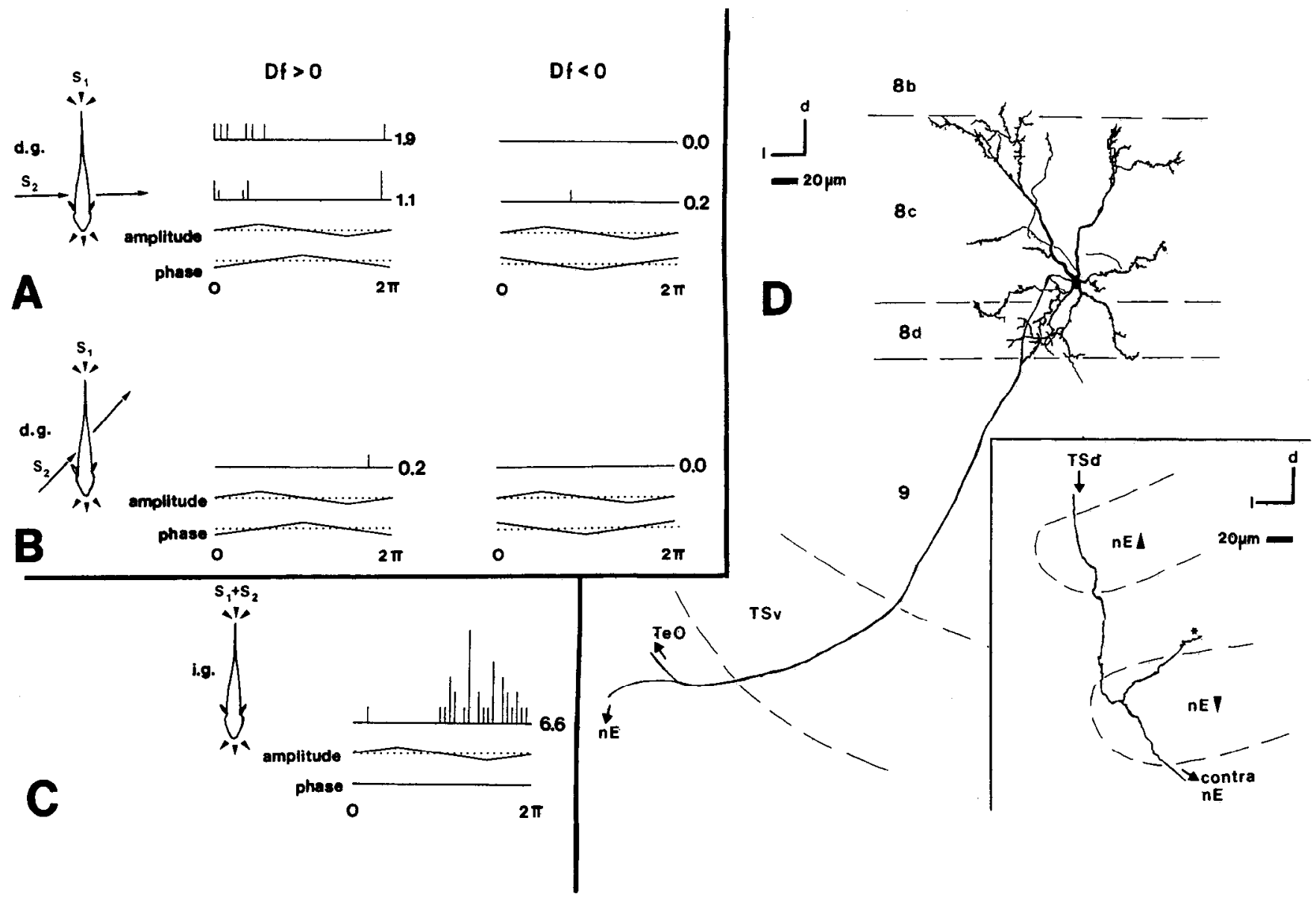

Figure 14. Responses of a phase-sensitive neuron in lamina $8 \mathrm{c}$ of the torus semicircularis dorsalis $(T S d)$. The EOD mimic $\left(S_{t}\right)$ is indicated in the drawings to the left (arrowheads show positive current flow). A jamming signal $\left(S_{2}\right)$ that was either $2 \mathrm{~Hz}$ higher $(D f>0)$ or $2 \mathrm{~Hz}$ lower $(D f<$ 0 ) than $\mathrm{S}_{1}$ was presented either through pairs of electrodes straddling the fish in different orientations (differential geometry, labeled $d . g$.; $A, B$ ) or through the same pair of electrodes used for delivering $S_{1}$ (identical geometry, labeled i.g.; C). The direction of the positive current flow for $S_{2}$ is indicated by the arrows in each of the drawings on the left. The spike rates are presented in histograms (bin width, $10 \mathrm{msec}$ ) covering one beat cycle of the interfering signals $S_{1}$ and $S_{2}$ and plotted above the amplitude and phase functions. The numbers to the right of each spike histogram are the relative numbers of spikes per beat cycle. For details of data presentation, see Heiligenberg and Rose (1985). $A$ and $B$, The neuron responded best to a jamming signal with a frequency higher than that of $S_{1}$ (amplitude rise combined with phase lag) and that was presented perpendicular to the fish's body axis (two replications of experiment in $A$ compared with $B$ ). $C$, Under identical geometry, when the interfering signal contained no phase information, the neuron responded to a decrease in amplitude and is thus classified as an I-unit. $D$, The soma of this neuron was located at the ventral border of lamina $8 \mathrm{c}$. The heavily spined dendrites extended up to the dorsal boundary of lamina $8 \mathrm{c}$ and spread into lamina $8 \mathrm{~d}$. The axon split into two collaterals after it left the torus. The thicker collateral projected to the optic tectum, and the thinner branch headed approximately $600 \mu \mathrm{m}$ rostrally toward the ipsilateral nucleus clcctrosncnsorius (inset, $n E$ ), passed through the dorsal nucleus electrosensorius $(n E \uparrow)$, and divided into two further branches. One of them terminated just dorsal to the ipsilateral ventral nucleus electrosensorius $(n E \downarrow)$, and the remaining branch passed through the postoptic commissure over to the contralateral side where it was lost. TeO, tectum opticum; $T S v$, torus semicircularis ventralis.

erations, while the response to signals with negative frequency differences, that is, EOD accelerations, was left intact. The JAR could only be eliminated entirely by lesioning both PPn and SPPn bilaterally. Injections of APV, an antagonist of NMDAtype receptors, into the $P$ n revealed very similar results as lesions of the SPPn.

The second pathway also controls the resting frequency by the level of tonic activity of the SPPn. By lesioning the SPPn, this tonic activity was eliminated and the resting frequency decreased accordingly. In addition, the SPPn receives tonic inhibition (presumably from the ventral nucleus electrosensorius). This is suggested by the increase of the resting frequency after injection of the GABA $A_{A}$ antagonist bicuculline into the SPPn.

Effects of APV injections into the pacemaker of Eigenmannia similar to those described in the present report were already shown in an earlier study (Fig. 4 in Kawasaki and Heiligenberg, 1990). Still earlier results of APV injections into the pacemaker (Dye and Heiligenberg, 1987; Dye, 1988; Dye et al., 1989) suggested that the input from the PPn causing gradual EOD accelerations might be mediated exclusively by NMDA-type receptors. According to our present knowledge, this appears no longer tenable.

Recent tracer experiments in Eigenmannia demonstrated that the inputs to the pacemaker from the PPn-G/CP, which causes gradual EOD accelerations (Fig. 2), and from the SPPn (Fig. 2) are also spatially separated (Heiligenberg and Metzner, unpublished observations). The medullary pacemaker nucleus contains two classes of neurons, pacemaker cells and relay cells. The pacemaker cells are electrotonically coupled, fire synchronously, and drive the relay cells, which transmit each pacemaker 
command pulse to the spinal motor neurons of the electric organ (Bennett, 1971; Dye and Meyer, 1986). According to our observation of terminals labeled by the anterograde transport of choleratoxin, fibers from the SPPn synapse upon the somata of relay cells, much as in the case of Sternopygus (Keller et al., 1991a), while fibers from the PPn-G/CP appear to terminate more diffusely and probably on the dendrites of pacemaker cells (Heiligenberg and Metzner, unpublished observations).

NMDA-type receptors mediate predominantly the input from the SPPn and AMPA-type receptors mediate the input from the PPn-G/CP (see Fig. 2). The two classes of glutamate-receptor ion channels, the NMDA and the non-NMDA (AMPA) subtypes, therefore appear to be localized at different synapses within the pacemaker nucleus of Eigenmannia. This is in contrast to findings in slice preparations of the mammalian hippocampus, where NMDA and non-NMDA subtypes of receptors are colocalized at individual synapses (e.g., Bekkers and Stevens, 1989).

Bullock et al. (1972) and Bastian and Yuthas (1984) have already mentioned distinct differences between the time courses of EOD accelerations and decelerations of the JAR in Eigenmannia. The acceleration phase usually is faster and has a more jittery time course (which corresponds to time constants with greater SEMs). This is consistent with the prelesion time constants shown in Figures $3 C$ and $6 C$ (solid columns). Bastian and Yuthas (1984) suggested that quite different mechanisms might underlie the EOD acceleration and the EOD deceleration. While EOD accelerations might be controlled by excitatory neuronal processes, the deceleration may be due to a passive relaxation of the pacemaker activity due to removal of a tonic input. The results presented here support this notion and indicate that these two behavioral components of the JAR are even mediated by two different pathways.

In addition, Figure 2 shows the connection between the PPn-C and the relay cells of the pacemaker nucleus. This pathway controls the production of communication signals, so-called chirps (Kawasaki et al., 1988a; Hopkins, 1989; Kawasaki and Heiligcnbcrg, 1990). The connection between the PPn-C and the relay cells is mediated by AMPA-type receptors and can be selectively blocked by CNQX (Dye et al. 1989; Kawasaki and Heiligenberg, 1989; verified also by my own experiments).

While the anatomical connection between the dorsal nucleus electrosensorius (Fig. 2, nE $\uparrow$ ) and the PPn-G/CP is known (Keller et al., 1990; Zupanc and Heiligenberg, 1992; Heiligenberg and Metzner, unpublished observations), it was so far not possible to determine the connection between the ventral nucleus electrosensorius ( $\mathrm{nE} \downarrow)$ and the SPPn. Extracellular injections of HRP, choleratoxin, and Phaseolus lectin into these structures have not yet revealed any apparent connection pattern. Since the connection between the SPPn and the pacemaker nucleus was found only recently by using new and more sensitive tracer techniques, future studies using still better tracers may reveal the anatomical connection between the nucleus electrosensorius and the SPPn.

\section{No single class of final "recognition units" in sight}

Neurons within the PPn-G/CP were suggested to represent final, feature-extracting elements or "recognition neurons" for the control of JAR-related shifts of the pacemaker frequency (see Fig. 1, PPn-G; Kawasaki et al., 1988a; Rose et al., 1988; Heiligenberg, 1991). The excitation of these neurons due to negative frequency differences would accelerate the pacemaker, and the inhibition of these neurons in response to positive frequency differences would decelerate the pacemaker. The results of the experiments presented here, however, suggest that there is no single class of final recognition units. Instead of being controlled by a feature extracting system, which is strictly hierarchically organized, the motor control of the JAR follows a more democratically organized "push-pull" principle (Fig. 2): the excitation of neurons in the PPn-G/CP in response to negative frequency differences is now assumed to accelerate the firing rate of pacemaker cells and thus to "push" the EOD frequency up, while the inhibition of neurons in the SPPn due to positive frequency differences should reduce their tonic excitation of the pacemaker and therefore "pull" the EOD frequency down. Although the SPPn neurons synapse upon the relay cells of the pacemaker nucleus, the strong electrotonic coupling between relay cells and pacemaker cells ensures that the SPPn controls the pacemaker in its entirety.

Nevertheless, extracellular recordings from neurons within the PPn-G demonstrated a decrease in activity below the spontaneous level during the presentation of positive frequency differences (Rose et al., 1988). The reduction, however, was rather small, and excitation in response to negative frequency differences usually dominated (Rose et al., 1988; G. J. Rose and M. Kawasaki, personal communication). How can this "inhibition" of cells in the PPn-G then be interpreted? It is known from pharmacological studies that the dorsal and ventral portions of the nucleus electrosensorius receive reciprocal GABAergic inhibition (Keller and Heiligenberg, 1989; see Fig. 2, nE $\uparrow$ and $n E \downarrow$ ). Whether this reciprocal inhibition is mediated by direct or indirect connections between the dorsal and the ventral nucleus electrosensorius is not known. Positive frequency differences, therefore, cause not only an excitation of the ventral nucleus electrosensorius but also an inhibition of the dorsal nucleus electrosensorius. This inhibitory "side effect" could account for the "inhibition" observed in neurons of the PPn-G: tonic activity of neurons in the dorsal nucleus electrosensorius would maintain the spontaneous activity level of cells in the PPn-G when no jamming stimulus is present. Since positive frequency differences excite neurons in the ventral nucleus electrosensorius, they should also reduce the spontaneous activity level of neurons in the dorsal nucleus electrosensorius, which in turn should lower the activity of cells in the PPn. Thus, no direct inhibitory input to the PPn-G would be required. This would also explain why we were unable to find any response to the injection of GABA or glycine in this area (see also Keller and Heiligenberg, 1989).

\section{Unsolved mysteries}

The flow diagram of the motor control of the JAR shown in Figure 2 summarizes major findings of the present study. Lesions of the SPPn, for example, leave the reciprocal connection between dorsal ( $\mathrm{nE} \mid$ ) and ventral nucleus electrosensorius ( $\mathrm{nE} \downarrow$ ) intact. As a consequence, stimulation of the ventral nucleus electrosensorius by jamming stimuli with positive frequency differences should still inhibit the dorsal nucleus electrosensorius and thereby lower the activity of the PPn (see discussion above), which in turn should reduce the tonic activity of the pacemaker. This indirect inhibitory effect could account for the slower EOD accelerations observed after lesions of the SPPn (Fig. 6C).

On the other hand, some results of lesions of the PPn are still puzzling. The inhibition of the ventral nucleus electrosensorius 
by the dorsal nucleus electrosensorius and the tonic inhibition of the SPPn by the ventral nucleus electrosensorius would suggest that, after lesions of the PPn, the resting frequency of the pacemaker and the baseline frequency during JAR should rise in response to jamming signals that stimulate the dorsal nucleus electrosensorius: increased activity of the dorsal nucleus electrosensorius should increase the inhibitory input to the ventral nucleus electrosensorius, which in turn should reduce its tonic inhibition of the SPPn and thus cause an increase in the activity of the SPPn. As a consequence, the pacemaker frequency should rise. After lesions of the PPn, however, the initial presentation of jamming stimuli with a negative frequency difference instead lowered the EOD frequency (Fig. 3B, white stars). The time courses of EOD accelerations after the lesion were even significantly slower than passive accelerations (i.e., the recovery from a previous decrease below the resting frequency; Fig. $3 C$ ). A detailed, quantitative analysis of the dynamics of the pacemaker and its various inputs will be required to explain such apparent inconsistencies.

\section{The role of the sublemniscal prepacemaker nucleus in other gymnotiform fish}

The SPPn is found in all gymnotiform fish investigated so far (Keller et al., 1991b). However, its function appears to differ among species. As demonstrated here, it appears to be involved in the motor control of the JAR in Eigenmannia by mediating the EOD deceleration caused by jamming stimuli with a positive frequency difference. In Sternopygus, a genus with a low-frequency signal, and in Hypopomus, a genus with pulse-type EODs, the stimulation of the SPPn with L-glutamate causes a sustained depolarization of the relay cells in the pacemaker nucleus, which leads to EOD interruptions (Keller et al., 1991a; Heiligenberg, personal communication) used in the context of social communication. Much as in Eigenmannia, the input from the SPPn to the pacemaker nucleus is mediated by NMDA-type receptors and can be blocked by APV. Neither Sternopygus nor Hypopomus shows a JAR similar to the JAR in Eigenmannia (Bullock, 1969). Fish of the genus Apteronotus, a fish with highfrequency wave-like EODs, increase their EOD frequency in response to negative frequency differences but are unable to lower their EOD frequency below the resting frequency (Bullock et al., 1972). Stimulation of the SPPn in Apteronotus causes gradual rise of the pacemaker frequency, which can be so high as to desynchronize and stop the pacemaker, leading to EOD interruptions observed in the context of courtship (Heiligenbcrg and Metzner, unpublished observations). Although the SPPn of Apteronotus is also exposed to the inhibitory influence of the ventral nucleus electrosensorius (Keller, 1989), Apteronotus obviously cannot lower its EOD frequency below the resting level since its SPPn is normally inactive.

\section{References}

Bastian J, Heiligenberg W (1980a) Neural correlates of the jamming avoidance response in Eigenmannia. J Comp Physiol 136:135-152.

Bastian J, Heiligenberg W (1980b) Phase sensitive midbrain neurons in Eigenmannia: neural correlates of the jamming avoidance response. Science 209:828-831.

Bastian J, Yuthas J (1984) The jamming avoidance response of $\mathrm{Ei}$ genmannia: properties of a diencephalic link between sensory processing and motor output. J Comp Physiol [A] 154:895-908.

Bekkers JM, Stevens CF (1989) NMDA and non-NMDA receptors are co-localized at individual excitatory synapses in cultured rat hippocampus. Nature 341:230-233.
Bennett MVL (1971) Electric organs. In: Fish physiology (Hoar WS, Kandall DJ, eds), pp 493-574. New York: Academic.

Bullock TH (1969) Species differences in effect of electroreceptor input on electric organ pacemakers and other aspects of behavior in electric fish. Brain Behav Evol 2:85-118.

Bullock TH, Hamstra RH, Scheich H (1972) The jamming avoidance response of high-frequency electric fish, I and II. J Comp Physiol 77: $1-48$.

Carr CE, Maler L (1985) A Golgi study of the cell types of the dorsal torus semicircularis of the electric fish Eigenmannia: functional and morphological diversity in the midbrain. J Comp Neurol 235:207240.

Carr CE, Maler L, Heiligenberg W, Sas E (1981) Laminar organization of the afferent and efferent systems of the torus semicircularis of gymnotiform fish: morphological substrates for parallel processing in the electrosensory system. J Comp Neurol 203:649-670.

Carr CE, Heiligenberg W, Rose GJ (1986a) A time-comparison circuit in the electric fish midbrain. I. Behavior and physiology. I Neurosci 6:107-119.

Carr CE, Maler L, Taylor B (1986b) A time-comparison circuit in the electric fish midbrain. II. Functional morphology. J Neurosci 6:13721383.

Dye JC (1988) An in vitro physiological preparation of a vertebrate communicatory behavior: chirping in the weakly electric fish, Apteronotus. J Comp Physiol [A] 163:445-458.

Dye JC, Heiligenberg W (1987) Intracellular recording in the medullary pacemaker nucleus of the weakly electric fish, Apteronotus, during modulatory behaviors. J Comp Physiol [A] 161:187-200.

Dye JC, Meyer JH (1986) Central control of the electric organ discharge in weakly electric fish. In: Electroreception (Bullock TH, Heiligenberg W, eds), pp 71-102. New York: Wiley.

Dye JC, Heiligenberg W, Keller C, Kawasaki M (1989) Different classes of glutamate receptors mediate distinct behaviors in a single brainstem nucleus. Proc Natl Acad Sci USA 86:8993-8997.

Hagedorn M, Heiligenberg W (1985) Court and spark: electric signals in the courtship and mating of gymnotoid electric fish. Anim Behav 33:254-265.

Heiligenberg W (1973) Electrolocation of objects in the electric fish Eigenmannia (Rhamphichthyidae, Gymnotoidei). J Comp Physiol 87:137-164.

Heiligenberg W (1991) Neural nets in electric fish. Cambridge, MA: MIT Press.

Heiligenberg W, Kawasaki M (1992) An internal current source yiclds immunity of electrosensory information processing to unusually strong jamming in electric fish. J Comp Physiol [A] 171:309-316.

Heiligenberg W, Ruse GJ (1985) Phase and amplitude computations in the midbrain of an electric fish: intracellular studies of neurons participating in the jamming avoidance response of Eigenmannia. J Neurosci 5:515-531.

Heiligenberg W, Rose GJ (1986) Gating of sensory information: joint computations of phase and amplitude data in the midbrain of the electric fish Eigenmannia. J Comp Physiol 159:311-324.

Heiligenberg W, Baker C, Matsubara J (1978) The jamming avoidance response in Eigenmannia revisited: the structure of a neuronal democracy. J Comp Physiol 127:267-286.

Heiligenberg W, Finger T, Matsubara J, Carr CE (1981) Input to the medullary pacemaker nucleus in the weakly electric fish, Eigenmannia (Sternopygidae, Gymnotiformes). Brain Res 211:418-423.

Heiligenberg W, Keller CH, Metzner W, Kawasaki M (1991) Structure and function of neurons in the complex of the nucleus electrosensorius of the gymnotiform fish Eigenmannia: detection and processing of electric signals in social communication. J Comp Physiol [A] 169: 151-164.

Hopkins CD (1974a) Electric communication in fish. Am Sci 62:426437.

Hopkins CD (1974b) Electric communication: functions in the social behavior of Eigenmannia virescens. Behavior 50:270-305.

Hopkins CD (1988) Neuroethology of electric communication. Annu Rev Neurosci 11:497-535.

Kawasaki M, Heiligenberg W (1988) Individual prepacemaker neurons can modulate the medullary pacemaker of the gymnotiform electric fish, Eigenmannia. J Comp Physiol [A] 162:13-21.

Kawasaki M, Heiligenberg W (1990) Different classes of glutamate receptors and GABA mediate distinct modulations of a neuronal 
oscillator, the medullary pacemaker of a gymnotiform electric fish. J Neurosci 10:3896-3904.

Kawasaki M, Maler L, Rose GJ, Heiligenberg W (1988a) Anatomical and functional organization of the prepacemaker nucleus in gymnotiform electric fish: the accommodation of two behaviors in one nucleus. J Comp Neurol 276:113-131.

Kawasaki M, Rose GJ, Heiligenberg W (1988b) Temporal hyperacuity in single neurons of electric fish. Nature 336:173-176.

Keller CH (1988) Stimulus discrimination in the diencephalon of $\mathrm{Ei}$ genmannia: the emergence and sharpening of a sensory filter. J Comp Physiol [A] 162:747-757.

Keller CH (1989) A sensory-motor interface in weakly electric gymnotiform fishes. PhD thesis, University of California at San Diego.

Keller CH, Heiligenberg (1989) From distributed sensory processing to discrete motor representations in the diencephalon of the electric fish, Eigenmannia. J Comp Physiol [A] 64:565-576.

Keller CH, Maler L, Heiligenberg W (1990) Structural and functional organization of a diencephalic sensory-motor interface in the gymnotiform fish, Eigenmannia. J Comp Neurol 293:347-376.

Keller CH, Kawasaki M, Heiligenberg W (1991a) The control of pacemaker modulations for social communication in the weakly electric fish Sternopygus. J Comp Physiol 169:441-450.

Keller CH, Kawasaki M, Heiligenberg W, Kennedy G, Metzner W (1991b) A sublemniscal prepacemaker nucleus in gymnotiform fish depolarizes relay celis of the pacemaker nucleus via NMDA-type receptors. 21st Annual Meeting of the American Society for Neuroscience 560:8.

Maler L, Sas E, Rogers J (1981) The cytology of the posterior lateral line lobe of high-frequency weakly electric fish (Gymnotoidei): dendritic differentiation and synaptic specificity in a simple cortex. $J$ Comp Neurol 195:87-140.

Maler L, Sas E, Johnston S, Ellis W (1991) An atlas of the brain of the electric fish Apteronotus leptorhynchus. J Chem Neuroanat 4:138 .

Metzner W (1992) The sublemniscal prepacemaker (SPPn) modulates the frequency of the medullary pacemaker nucleus in the gymnotiform fish, Eigenmannia. 22nd Annual Meeting of the American Society for Neuroscience 149:9.

Metzner W, Heiligenberg W (1991a) The coding of signals in the electric communication of the gymnotiform fish Eigenmannia: from electroreceptors to neurons in the torus semicircularis of the midbrain. J Comp Physiol [A] 169:135-150.

Metzner W, Heiligenberg W (1991b) Midbrain and diencephalic links within the neuronal network underlying the jamming avoidance response in Eigenmannia. 21 st Annual Meeting of the American Socicty for Neuroscience 560:10.

Rose GJ, Heiligenberg W (1985a) Structure and function of electrosensory neurons in the torus semicircularis of Eigenmannia: morphological correlates of phase and amplitude sensitivity. J Neurosci 5:2269-2280.

Rose GJ, Heiligenberg W (1985b) Temporal hyperacuity in the electric sense of fish. Nature 318:178-180.

Rose GJ, Heiligenberg W (1986) Limits of phase and amplitude sensitivity in the torus semicircularis of Eigenmannia. J Comp Physiol [A] 159:813-822.

Rose GJ, Kawasaki M, Heiligenberg W (1988) "Recognition units" at the top of a neuronal hierarchy? J Comp Physiol [A] 162:759-772.

Scheich H, Bullock TH (1974) The role of electroreceptors in the animal's life. II. The detection of clectric fields from electric organs. In: Handbook of sensory physiology, Vol III/3 (Fessard A, ed), pp 201-256. Berlin: Springer.

Szabo T, Heiligenberg W, Ravaille-Veron M (1989) HRP labeling and ultrastructural localization of prepacemaker terminals within the medullary pacemaker nucleus of the weakly electric gymnotiform fish Apteronotus leptorhynchus. J Comp Neurol 824:169-173.

Watanabe A, Takeda K (1963) The change of discharge frequency by AC stimulus in a weakly electric fish. J Exp Biol 40:57-66.

Zupanc GKH, Heiligenberg W (1992) The structure of the diencephalic prepacemaker nucleus revisited: light microscopic and ultrastructural studies. J Comp Neurol 323:558-569. 